\title{
THE COSMIC SUBMILLIMETER BACKGROUND AS A POSSIBLE SIGNATURE OF THE INITIAL BURST OF GALAXY FORMATION
}

\author{
S. DJorgovski ${ }^{1}$ AND Nicholas WeIR \\ Division of Physics, Mathematics, and Astronomy, California Institute of Technology \\ Received 1989 May 17; accepted 1989 September 11
}

\begin{abstract}
We propose a heuristic model for the origin of the cosmic submillimeter background (SMB), reported by the Nagoya-Berkeley collaboration. The SMB is interpreted as a direct signature of an epoch of (initial) galaxy formation at $z \sim 10-15$. The sources of the SMB are proposed to be dust-shrouded starburst protogalaxies, similar to the luminous IRAS galaxies at low redshifts. We interpret them as the progenitors of old stellar populations at low redshifts, ellipticals, bulges, and stellar components of the halos. The derived redshift of the galaxy formation is directly dependent on the dust temperatures assumed for these objects. The corresponding look-back times are $\sim 11.5 h_{75}^{-1} \mathrm{Gyr}$ for $\Omega_{0}=0.1$, or $\sim 8.5 h_{75}^{-1} \mathrm{Gyr}$ for $\Omega_{0}=1$. The star formation history in an element of comoving volume was assumed to be a Gaussian in the rest frame, but this form is not critical for the models. Model spectra of the SMB were computed for the values of cosmological density parameter $\Omega_{0}=$ 0.1 and 1 , and the dust emissivity index $n=1$ and 2. The largest allowed time scales for the star formation in these models (expressed as the FWHM of the luminosity history) are in the range FWHM $\sim 0.2-0.6$ Gyr for the low-density models $\left(\Omega_{0}=0.1\right)$; for the high-density models $\left(\Omega_{0}=1\right)$, the allowed widths are about a factor of 2 lower. These widths are comparable to, or slightly larger than, the free-fall times for normal galaxies. In order not to overproduce the baryonic mass density, it is necessary that the initial mass function (IMF) in these starbursts is biased toward high-mass stars; however, a substantial range in the IMF parameters is allowed. This postulated population of protogalaxies may be an important contributor to the diffuse soft X-ray background. Leaked (unobscured) starlight from these objects may give rise to a near-infrared background, at about the level detected by Matsumoto, Akiba, and Murakami. The predicted surface density of protogalaxies would be in the range $\sim 10-100 \operatorname{arcsec}^{-2}$, which is consistent with all relevant anisotropy measurements available at this time. The model also predicts that a considerable fraction of the mass density in the bulge and halo of our Galaxy would be provided by old white dwarfs, which may be detectable in deep surveys (a similar prediction was already made by Silk). Spectroscopic signatures of this population may be detectable with future space missions, e.g., with $S I R T F$ or $L D R$, and possibly also from the ground in the near-infrared and millimeter/submillimeter regions.
\end{abstract}

Subject headings: cosmic background radiation - cosmology - early universe - galaxies: formation radiation mechanisms

\section{INTRODUCTION}

Recently, the Nagoya-Berkeley collaboration reported the results of a measurement of the spectrum of the cosmic microwave background (CMBR) from a rocket-borne platform (Matsumoto et al. 1988). They found an "excess" emission in the submillimeter wavelength region, above the level expected from the CMBR and the interstellar dust; they argued that this submillimeter background (SMB) is not caused by any instrumental effect or any known Galactic source and that it is most likely cosmological in origin. If so, the SMB must be a signature of some important event or process in the early universe, because of its energetics: the energy density of the SMB is of the order of $20 \%$ of that of the CMBR. Whereas the Matsumoto et al. (1988) measurement still needs to be confirmed (for example, Halpern et al. 1988 found no evidence for the SMB excess, on the basis of balloon-borne experiments), the potential significance of the result warrants an inquiry in its possible origins. We note that the $T_{\mathrm{CMBR}}$ measurement at $1.32 \mathrm{~mm}$ by Meyer, Roth, and Hawkins (1989) is consistent with the Matsumoto et al. data.

As pointed out, e.g., by Bond, Carr, and Hogan (1986), there is a large gap in our empirical knowledge about the universe

${ }^{1}$ Alfred P. Sloan Foundation fellow. between the epoch corresponding to the most distant quasars known, at $z \sim 4-5$, and the epoch of decoupling (the CMBR photosphere, at $z \sim 1000$ ). Many important processes are likely to have happened in that interval; e.g., the early stages, or perhaps even the bulk, of galaxy formation and the initial chemical enrichment, development of the large-scale structure, the appearance of quasars, etc. Most of these processes could have left imprints on the CMBR (see, e.g., the reviews by Sunyaev and Zel'dovich 1980; Kaiser and Silk 1986; Partridge 1987). The generic expectation is that the energy released in these epochs would now be observable in the infrared and submillimeter regions due to redshifting, and possibly also due to reprocessing by dust at large redshifts.

The two most likely mechanisms for production of the SMB were discussed by Hayakawa et al. (1987): emission by the dust heated by pregalactic stars or other sources, and Compton scattering of the CMBR, the former mechanism providing a better fit to the data. Lacey and Field (1988) find that the Compton mechanism probably fails on energetics grounds, but see also Rephaeli and Smoot (1988), Taylor and Wright (1989), or Bartlett and Silk (1989) for related discussions. Another proposed mechanism is that the SMB is produced by the decay of hypothetical relic particles (Kawasaki and Sato 1987); however, Raffelt, Dearborn, and Silk (1989), and Dar, Loeb, 
and Nussinov (1989) show that such an explanation may violate a variety of astrophysical constraints. It is also possible to interpret the data as the sum of a blackbody component (i.e., the CMBR as observed at longer wavelengths), and a graybody component caused by some as yet unspecified prerecombination process (Daly 1988). The Partridge (1987) "panda" mechanism is another possibility.

The scenario which received most attention to date involves energy generation at a high redshift, e.g., in pregalactic (Population III) stars, very massive objects, accreting pregalactic black holes, exploding cosmic strings, or some other exotic process, and its reprocessing by dust at some intermediate redshifts, e.g., $z>3$ (Carr 1987, 1989; Rowan-Robinson and Carr 1988; Hogan and Bond 1988; Silk 1988b; Adams et al. 1989). These works were developed in part from the earlier models by Negroponte (1986), McDowell (1986), and Bond, Carr, and Hogan (1986), which predate the discovery of the SMB by Matsumoto et al. The paper by Bond, Carr, and Hogan (1986) is particularly useful and provides the earlier references. The source of the energy is not really critical in this scheme, but it requires a somewhat ad hoc geometrical arrangement, in which the universe was opaque at the range of redshifts between the most distant quasars now observed (e.g., $z_{\text {dust }}>4$ ), and the primary sources $\left(z_{\text {dust }}<20-100\right.$ ?). Given such geometry, the scenario does lead naturally to a predicted spectrum very similar to the observed SMB. There is little or no information in the resulting spectrum about the redshift of the energy sources: the peak of the spectrum is determined by the redshift of the dust photosphere, $z_{\text {dust }}$. Adams et al. (1989) explored the radiation transfer and the energetics in this scenario and showed that energy released from stellar nucleosynthesis can account for the observed intensity of the SMB.

Here we explore a heuristic variant of the dust reradiation model. Instead of assuming some hypothetical or exotic process at a large redshift and an arbitrary geometry of the intervening dust, we attempt to explain the SMB in terms of objects and phenomena which we know exist at low redshifts, and which may well have high-redshift analogs. Specifically, we propose that the sources of the SMB are similar to the lowredshift, extremely luminous far-infrared galaxies (ELFs), like those detected with the IRAS satellite (Neugebauer et al. 1984). We tentatively identify such sources as protogalaxies, viz., progenitors of the present-day ellipticals and bulges, and perhaps also old disks. The SMB is thus interpreted as a signature of the epoch of initial galaxy formation (some galaxy formation, in particular the disks of spirals, or gas-rich dwarf systems, may well have extended to lower redshifts). Our model may also account for a large part of the diffuse, soft X-ray background.

\section{SOME PRELIMINARY CONSIDERATIONS}

We model the proposed high-redshift sources of the SMB according to the properties of low-redshift ELFs, well-studied IRAS starburst/merging galaxies (Houck et al. 1985; Soifer, Houck, and Neugebauer 1987, and references therein). We thus avoid the ambiguities of the geometry of dust, gas, and stars, optical depth, temperature gradients, reradiation processes, etc. We simply use these objects as ready-made, premixed building blocks for a shell in look-back time, or redshift, in which there is enhanced merging and starburst activity. The redshift of this shell, which we tentatively identify as the epoch of galaxy formation, is determined by the ratio of the peak wavelengths of the SMB and the energy distributions of our template ELFs. The extrapolation to large redshifts is critical for the quantitative aspects of our model; for example, the effective dust temperature may have been higher in the past, e.g., resulting from increased heating from the CMBR. Yet the basic idea still remains: we argue that ELFs provide a plausible empirical model for protogalaxies and that their hypothetical high-redshift counterparts are natural sources for the SMB. Since in our model the dust is clumped around the sources and the emergent radiation is mainly in the farinfrared, we assume that the universe is transparent to the SMB at all redshifts since its emission. This assumption alleviates the need for a detailed radiation transfer computation, which was already well explored by Bond, Carr, and Hogan (1986) and Adams et al. (1989).

A variety of morphological and other evidence suggests that ELFs are highly dissipative mergers (Joseph and Wright 1985; Joseph 1987; Soifer, Houck, and Neugebauer 1987, and references therein). It is generally believed that the primary energy sources of ELFs are dust-shrouded starbursts with characteristic time scales of $\sim 10-50$ million years (Rieke et al. 1980). Mergers often have been implicated in triggering starbursts (Larson and Tinsley 1978; Schweizer 1987, and references therein). It is also possible that for short periods of time ( $\sim 10^{5}-10^{6}$ yr per collision), a substantial part of their luminosity is derived from the kinetic energy of colliding or merging fragments (Harwit et al. 1987; see also Binney 1977). Whereas the release of binding energy may dominate the luminosity at selected times, the overall energy production integrated over $t \geq 10^{8} \mathrm{yr}$ is probably dominated by stellar nuclear burning. These are believed to be the generic processes of galaxy formation (Silk and Norman 1981; Silk 1987a, b).

The idea of interpreting the SMB as a result of dusty starbursts at large redshift has already been mentioned by McDowell (1988), Silk (1988b), Adams et al. (1989), and many others in the papers cited above, but it was not pursued in detail. One of the reasons for the neglect of this scheme may be the fact that the observed SMB is well-fitted by a singletemperature dust spectrum; there is not much space for intrinsic broadening. One of our goals is to explore the possible width of the epoch of galaxy formation which can be accommodated by the SMB data without violating any other astrophysical constraints, e.g., the overproduction of mass locked up in stars or stellar remnants or of the heavy elements.

As the first step in our analysis, we separate the spectrum of the SMB from the total intensity measurements as provided by Matsumoto et al. The crucial parameter is the assumed blackbody temperature of the CMBR and its uncertainty. Following the measurements and summaries by Crane et al. (1986), and Bersanelli et al. (1989), we adopt the value $T_{\mathrm{CMBR}}=2.74 \pm 0.04$ $\mathrm{K}$. The implied range contains the best available measurements of the $T_{\mathrm{CMBR}}$, and the error bar is conservative, but reasonable. Subtracting the appropriate CMBR contribution from the measurements by Matsumoto et al., we obtain the values listed in Table 1. Because of the uncertainties in subtracting of the galactic interstellar dust contribution, the values for channel 4 $(\lambda=262 \mu \mathrm{m})$ are upper limits only. The error bars shown incorporate both the measurement errors of the total intensity, quoted by Matsumoto et al., and the uncertainty of the $T_{\mathrm{CMBR}}$. Throughout this paper, we display the results both as $I_{v}$, which is useful in discussing various possible observational tests, and as $v I_{v}$, which gives a better impression of the energy contributions at different wavelengths and is often used in similar presentations. 
TABLE 1

ADOPTED FLUXES FOR THE SMB

\begin{tabular}{lrcc}
\hline \hline $\begin{array}{l}\text { Channel } \\
\text { Number }\end{array}$ & $\begin{array}{c}\lambda \\
(\mu \mathrm{m})\end{array}$ & $\begin{array}{c}v I_{v} \\
\left(10^{-11} \mathrm{~W} \mathrm{~cm}^{-2} \mathrm{sr}^{-1}\right)\end{array}$ & $\begin{array}{c}I_{v} \\
\left(10^{-4} \mathrm{Jy} \operatorname{arcsec}^{-2}\right)\end{array}$ \\
\hline $1 \ldots \ldots \ldots \ldots$ & 1160 & $0.76 \pm 0.53$ & $6.91 \pm 4.82$ \\
$2 \ldots \ldots \ldots \ldots$ & 709 & $2.12 \pm 0.38$ & $11.78 \pm 2.11$ \\
$3 \ldots \ldots \ldots \ldots$ & 481 & $1.42 \pm 0.15$ & $5.36 \pm 0.57$ \\
$4 \ldots \ldots \ldots \ldots$ & 262 & $<0.39$ & $<0.80$ \\
\hline
\end{tabular}

Because of the sparse sampling (three data points and one upper limit), some interpolating function is needed. As is common in all models where the thermal emission from dust is considered, we use a family of functions:

$$
I_{v}=\text { const } \times v^{n} B_{v}(T),
$$

where $B_{v}(T)$ is the Planck function, and the dust emissivity index $n$ is generally found to be in the range 1-2 (Thronson and Harper 1979; Telesco and Harper 1980; Draine and Lee 1984; Emerson 1988; Helou 1989). We use two cases, $n=1$, and $n=2$, in order to cover the plausible range. The overall shape of the emergent spectrum from a dust-shrouded star-forming region is dependent in a complex manner on the internal geometry of the system, e.g., distribution of the heating sources, optical depth of the obscuring dust, geometrical and chemical properties of the dust grains, etc. (Draine and Lee 1984; Wright 1987; Emerson 1988; Hawkins and Wright 1988).

We bypass these problems by fitting the dust spectra (eq. [1]) to the well-observed nearby objects, viz., M82 and Arp 220. M82 is the nearest example of a luminous infrared starburst galaxy (Rieke et al. 1980). Arp $220=$ IC 4553 is a prototypical ELF galaxy (Soifer et al. 1984; Emerson et al. 1984; Rieke et al. 1985; Joy et al. 1986; Smith et al. 1988). In order to probe the dust emission peak, we use the homogeneous IRAS measurements for M82 from Rice et al. (1988), and for Arp 220 from Soifer et al. (1984), plus the $350 \mu \mathrm{m}$ point from Emerson et al. (1984). We treat the $12 \mu \mathrm{m} I R A S$ points as upper limits, since they also include a contribution from the unreddened stars. The data and the models are shown in Figure 1. In the $n=1$ case, we obtain the best-fit, redshift-corrected temperatures of $62.8 \mathrm{~K}$ for M82 and $54.1 \mathrm{~K}$ for Arp 220; biasing toward Arp 220, as the more typical ELF, we adopt $T_{n=1}=55$ $\mathrm{K}$. In the $n=2$ case, we obtain best-fit temperatures of $52.5 \mathrm{~K}$ for M82 and 43.5 K for Arp 220; we adopt $T_{n=2}=45 \mathrm{~K}$.

It is possible that the properties of our hypothetical highredshift sources of the SMB were sufficiently different that the emergent spectrum had a different shape or temperature. For example, the observed IRAS spectra of M82 and Arp 220 are sufficiently narrow that they can be fitted by singletemperature dust spectra. If a balanced mix of dust of different temperatures was present, any additional redshift broadening would make it very difficult to fit the SMB data. It is, however, reassuring that the thermal dust spectra from a variety of lowredshift sources show a very little variation, and that in low-z ELFs a single dust component usually dominates (Helou 1989), but the relevant physical and chemical circumstances could well have been different at $z \sim 10$. We cannot make any reliable estimate of such variations, and our models are meant to be illustrative rather than definitive. This is a potential difficulty common to all dust reradiation models of the SMB. With that caveat, we proceed.

Fits to the Matsumoto et al. SMB data, in the singletemperature approximation, give $T_{\mathrm{SMB}}=4.25 \mathrm{~K} \times\left(1+z_{\mathrm{SMB}}\right)$ for the $n=1$ case and $T_{\mathrm{SMB}}=3.59 \mathrm{~K} \times\left(1+z_{\mathrm{SMB}}\right)$ for the $n=2$ case. This suggests that the typical redshifts for the sources are $z_{\text {SMB }} \sim 12$, assuming our template spectra. This redshift range is also fully consistent with the general arguments presented by Draine and Shapiro (1989).

We also need to make a choice of cosmology. We will use simple, $\Lambda=0$ Friedman models, with the Hubble parameter $H_{0}=75 \mathrm{~km} \mathrm{~s}^{-1} \mathrm{Mpc}^{-1}$, corresponding to the Hubble time $t_{H}=13.04$ Gyr. The scaling of quantities dependent on $H_{0}$ is accounted for with the parameter $h_{75} \equiv H_{0} /\left(75 \mathrm{~km} \mathrm{~s}{ }^{-1}\right.$ $\left.\mathrm{Mpc}^{-1}\right)$. The closure density is then $\rho_{\text {crit }}=1.057 \times 10^{-29} h_{75}^{2} \mathrm{~g}$
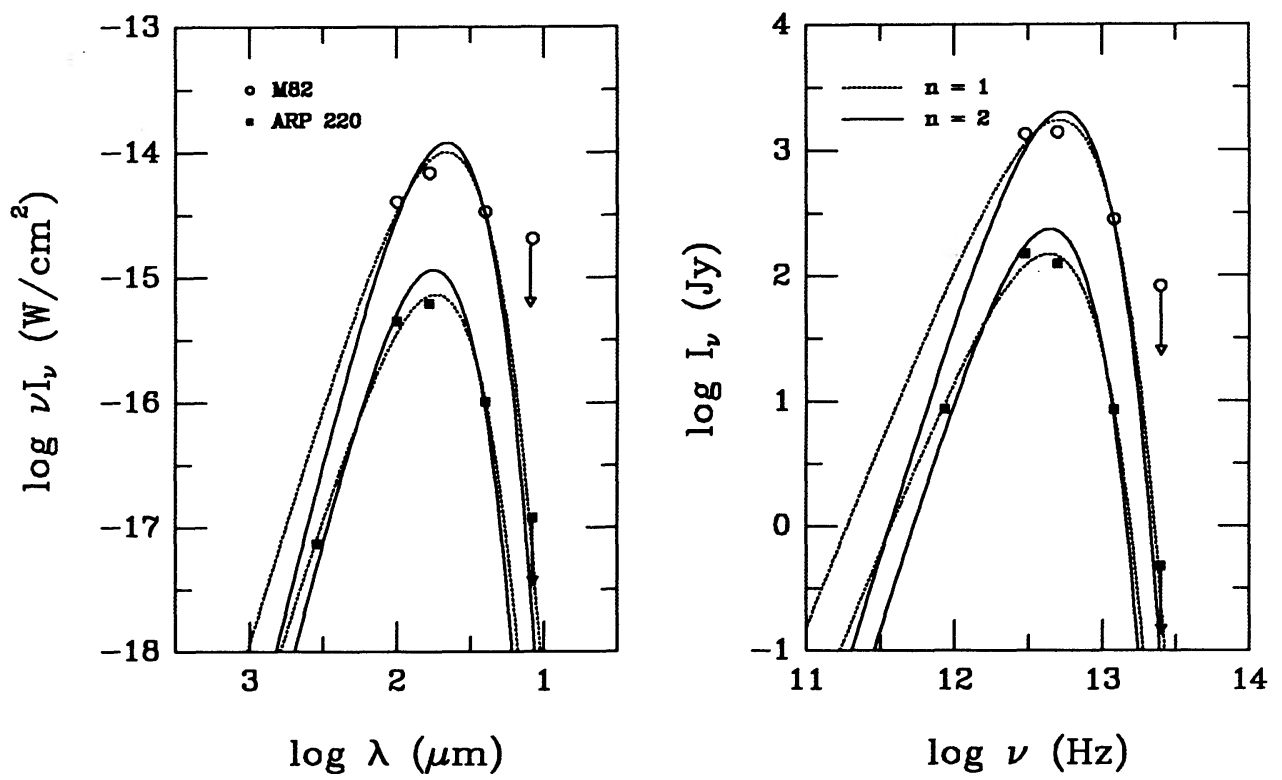

Fig. 1.-Data on the template starburst galaxies M82 (open circles) and Arp 220 (solid squares), from the references given in the text. The fits for $n=1$ (dotted line)

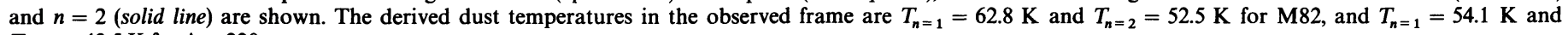
$T_{n=2}=43.5 \mathrm{~K}$ for Arp 220 . 
$\mathrm{cm}^{-3}=1.561 \times 10^{11} h_{75}^{2} M_{\odot} \mathrm{Mpc}^{-3}$. We use two possible values of the density parameter, $\Omega_{0}=0.1$ and $\Omega_{0}=1$, which cover a reasonable range of the observed values and theoretical prejudices (see Peebles 1986), and we consider the lower value to be the more realistic one. The baryonic contribution to density, $\boldsymbol{\Omega}_{b}$, is also of some interest. The classical (uniform density big bang necleosynthesis) upper limit, assuming $T_{\mathrm{CMBR}}=2.74 \mathrm{~K}$, and the photon-to-baryon parameter $\eta_{10}=$ 10 , is $\Omega_{b}=0.00656 h_{75}^{-2}$ (Yang et al. 1984; Boesgaard and Steigman 1985). However, in the nonhomogeneous big bang nucleosynthesis models, considerably larger values may be possible, up to $\Omega_{b}=1$ (Applegate and Hogan 1985; Sale and Mathews 1986; Applegate, Hogan, and Scherrer 1987; Alcock, Fuller, and Mathews 1987; Malaney and Fowler 1988). A plausible estimate may be $\Omega_{b} \simeq 0.1$.

\section{ENERGETICS, YIELDS, AND STARBURSTS}

Perhaps the most surprising and interesting property of the SMB is its relatively large energy density, From the fits of the thermal dust emission spectra and the models described below to the data points from Table 1, we derive its energy density, $u_{\mathrm{SMB}} \simeq(9 \pm 2) \times 10^{-14}$ ergs $\mathrm{cm}^{-3}$. For comparison, the energy density of the CMBR, assuming $T_{\mathrm{CMBR}}=2.74 \mathrm{~K}$, is $u_{\mathrm{CMBR}}=4.26 \times 10^{-13} \mathrm{ergs} \mathrm{cm}^{-3}$. The two proposed mechanisms of energy generation in our model, the release of binding energy during galaxy formation and stellar nuclear burning, should be capable of producing the required amounts of energy density. Here we discuss their relative importance. A related discussion is presented by Daly and Turner (1988).

The contribution from stellar sources can be estimated as follows. The energy released in transforming hydrogen into helium is $\sim 7 \mathrm{MeV}$ nucleon $^{-1}$. Further transformation up to iron yield $\sim 1 \mathrm{MeV}$ nucleon $^{-1}$ more. The energy generated per unit mass is thus $\sim 0.007 c^{2}$, and the resulting energy density is

$$
u_{\mathrm{nuc}} \simeq 0.007 f_{x} \Omega_{*} \rho_{\mathrm{crit}} c^{2},
$$

where $f_{x}$ is the fraction of the total mass of the fuel available, which is not greater than the primordial hydrogen fraction, $X \sim 0.75, \Omega_{*}$ is the fraction of the closure density in burnedout stars; we estimate $\Omega_{*} \leq 0.1$. The maximum energy density provided by the stellar nucleosynthesis is thus $u_{\text {nuc }} \sim 5.0$ $\times 10^{-12} h_{75}^{2}$ ergs $\mathrm{cm}^{-3}$, which is more than sufficient to account for the SMB, even with a large inefficiency factor.

The binding energy density of galaxies can be estimated from the mean mass density and the characteristic onedimensional internal velocities, $V_{g}$, from the virial formula:

$$
u_{\text {bind }} \simeq \frac{3}{2} V_{g}^{2} \rho_{\text {gal }},
$$

where $\rho_{\text {gal }}$ is the mean mass density associated with galaxies, which can be represented as $\rho_{\text {gal }}=\Omega_{g} \rho_{\text {crit }}$, where $\Omega_{g}$ is the fraction of the closure density in galaxies. The typical values of the $V_{g}$, i.e., rotational velocity of disks, or the projected velocity dispersion of spheroids, are $\sim 200 \mathrm{~km} \mathrm{~s}^{-1}$. If we make an estimate $\Omega_{g} \sim 0.1$, then $\rho_{\text {gal }} \sim 10^{-30} h_{75}^{2} \mathrm{~g} \mathrm{~cm}^{-3}$, and $u_{\text {bind }} \simeq 6$ $\times 10^{-16} h_{75}^{2}$ ergs $\mathrm{cm}^{-3}$. Alternatively, we can use the measured luminosity density. From Kirshner et al. (1983), we obtain the luminosity density in the $V$ band $u_{L V}=1.8 \times 10^{8} h_{75} L_{\odot}$ $\mathrm{Mpc}^{-3}$. From de Lapparent, Geller, and Huchra (1989), we obtain the luminosity density in the $B$ band $u_{L B}=(1.5 \pm 0.4)$ $\times 10^{8} h_{75} L_{\odot} \mathrm{Mpc}^{-3}$. Using the difference between the typical $B-V$ colors of galaxies $(\sim 0.85-0.9 \mathrm{mag})$ and of the Sun $(0.65$ mag), we convert the later into $u_{\mathrm{LV}}=(1.8 \pm 0.5) \times 10^{8} h_{75} L_{\odot}$ $\mathrm{Mpc}^{-3}$, in an excellent agreement with the older Kirshner et al.
(1983) result. The mass density is then $\rho_{\text {gal }}=u_{L V}(M / L)_{V}$, where $(M / L)_{V}$ is the typical galactic mass-to-light ratio at large radii in solar $V$ band units. The $(M / L)_{V}$ is believed to be in the range of 10-200 for individual galaxies or compact groups; assuming $(M / L)_{V} \sim 50$, we obtain $\rho_{\mathrm{gal}} \sim 10^{10} h_{75} M_{\odot} \quad \mathrm{Mpc}^{-3} \simeq 7$ $\times 10^{-31} h_{75} \mathrm{~g} \mathrm{~cm}^{-3}$, and $u_{\text {bind }} \simeq 4 \times 10^{-16} h_{75}$ ergs $\mathrm{cm}^{-3}$. Thus, the release of the binding energy of galaxies is an insufficient source for the SMB, by about two orders of magnitude, and it is very small compared to the energy which is in principle available from stellar burning. Moreover, some of the binding energy may have been released at larger redshifts $(z>20-30)$ by inverse Compton cooling against the CMBR (Rees and Ostriker 1977; White and Rees 1978; Efstathiou and Silk 1983, and references therein).

In order to make some quantitative estimates about the mass consumption and energy production in starburst from stellar burning, and to explore the dependence of relevant quantities on the initial mass function (IMF), we constructed simple starburst models as follows. We used IMFs in the standard power-law form, $\Psi(M)=S \times M^{-(1+x)}$, for $M_{\mathrm{L}} \leq M \leq$ $M_{\mathrm{U}}$, and $\Psi(M)=0$ outside that range. The scaling factor $S$ was determined by the requirement that the star formation rate SFR $=1 M_{\odot} \mathrm{yr}^{-1}$, for the duration of the star formation era, $0 \leq t \leq \tau$, and SFR $=0$ for $t>\tau$. This is not to imply that the observed ELFs or our hypothetical SMB sources have such low SFR; it is simply an easy-to-scale number of convenience, which drops out of the relevant calculations anyway (see below).

The models are thus completely specified by the four parameters, IMF slope $x, M_{\mathrm{L}}, M_{\mathrm{U}}$, and $\tau$. For $x$, we use $0.5,1.35$ (Salpeter IMF), and 2.5; for $M_{\mathrm{L}}, 0.1$ and $1 M_{\odot}$; for $M_{\mathrm{U}}, 10,20$, and $100 M_{\odot}$; and for $\tau, 10,20,30,50,100$, and $200 \mathrm{Myr}$. Models were run with all possible combinations of these parameters. The lifetimes of stars of different mass were taken from the data tabulated by Scalo (1986). In order to compute the luminosity from the stars on the main sequence, we used the mass-luminosity relation given by Renzini and Buzzoni (1986), in the form $L_{\mathrm{bol}}(M)=10^{a} \times M^{\alpha}$, where for the mass ranges $\quad M>4, \quad 1.4<M \leq 4, \quad 0.57<M \leq 1.4, \quad$ and $0.1<M \leq 0.57$, the corresponding values of $(a, \alpha)$ are $(0.3998$, $3.4115)$, (0.1014, 3.8910), (-0.0336, 4.8152), and $(-0.5840$, 2.5540), and all masses and luminosities are in the solar units. In order to correct for the luminosity contributions of stars past the main sequence, we used the correction functions given by Renzini and Buzzoni (1986) for the three IMF slopes. However, in all models, on the time scales of interest to us ( $\leq 10^{8}$ yr or so), the luminosity is dominated by the upper main sequence. All stars with $M>8 M_{\odot}$ were assumed to explode as supernovae and to release $10^{51}$ ergs per event. In each model, we compute the luminosity as a function of time, $L_{\mathrm{bol}}(t)$, average luminosity, $\left\langle L_{\text {bol }}\right\rangle_{<t}$, and the cumulative energy released up to that time. The integration is done in steps of $1 \mathrm{Myr}$. Some representative luminosity histories are shown in Figures $2 a$ and $2 b$. The luminosity always increases up to $t=\tau$ and declines afterward; thus the FWHM of the luminosity history can be used as a characteristic time scale of the luminosity behavior of the starburst (note that $\tau$ is a characteristic time scale of the mass consumption, and whereas the two are mutually proportional, their exact relation depends on the IMF).

In order to relate the luminosities to masses processed into stars, we define the fuel consumption efficiency factor, $\Xi$ :

$$
\Xi=\left(\frac{\mathrm{SFR}}{M_{\odot} \mathrm{yr}^{-1}}\right)\left(\frac{L_{\mathrm{bol}}}{10^{11} L_{\odot}}\right)^{-1} \text {. }
$$



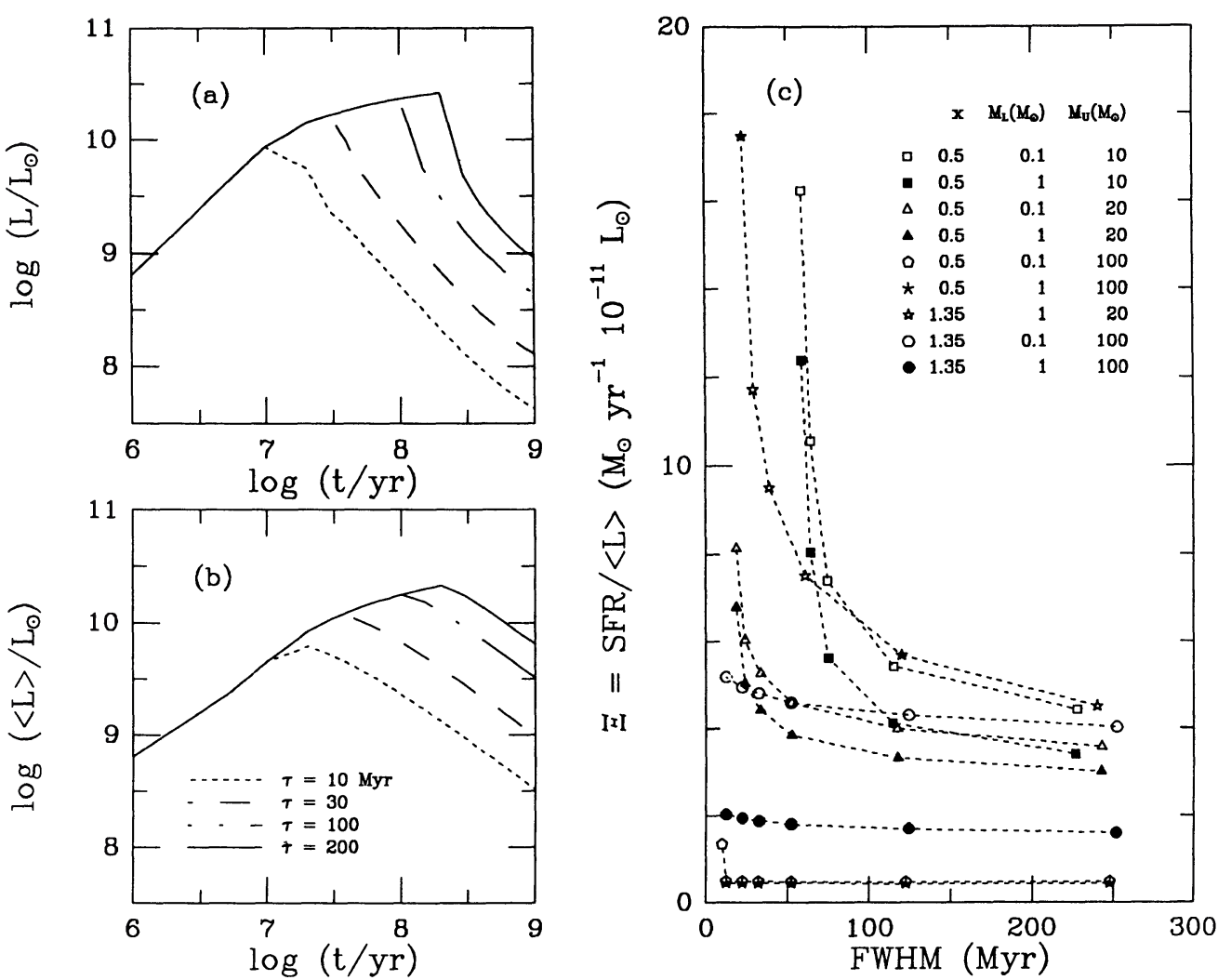

Fig. 2. (a) Luminosity histories of starburst models with the IMF determined by: $x=1.35, M_{\mathrm{L}}=0.1 M_{\odot}$, and $M_{\mathrm{U}}=20 M_{\odot}$. Different lines correspond to the different durations of the star formation, $\tau$, in Myr, as indicated. (b) Average luminosity histories of the same models as in $(a)$. (c) Dependence of the fuel consumption efficiency parameter, $\Xi$, on the FWHM of the luminosity history, in Myr, for different IMF parameters, coded with different symbols as indicated.

Clearly, $\Xi$ will depend on the IMF and the SFR history and will change in time. Operationally, we determine it in our models by using the mean bolometric luminosity for $t \leq 2 \tau$, and the mean SFR defined as the total mass of stars created by that time and divided by $2 \tau$. The choice of $t=2 \tau$ gives a fair estimate of the average energy production in the burst; the values of $\Xi$ determined at $t=\tau$ are typically about $10 \%$ higher. The values of $\Xi$ as functions of the FWHM of burst luminosity, $L(t)$, are plotted in Figure $2 c$ for several IMFs of interest. As we will argue below, the range of values of $\Xi$ which is preferred in our models (in order not to overproduce the cosmological density of the burned-out stars, $\Omega_{*}$ ) is $\Xi \leq 5$ or so. In general, the IMFs with the Salpeter slope or flatter satisfy this condition for all values of $\tau \geq 20 \mathrm{Myr}$, and $M_{\mathrm{U}} \geq 20$, if $M_{\mathrm{L}}=1 M_{\odot}$, and in most cases even if $M_{\mathrm{L}}=0.1 M_{\odot}$. Steeper IMFs, and in some cases IMFs with $x \leq 1.35$ and $M_{\mathrm{U}} \geq 10$, overproduce the mass.

Thus, we require that the IMF in starbursts which can generate the SMB is biased toward the more massive stars, or, perhaps more accurately, is not dwarf dominated. This can be accomplished through any of the three parameters which define the IMF: $x, M_{\mathrm{U}}$, or $M_{\mathrm{L}}$. Many authors propose that the IMF in starbursts is biased toward the high-mass stars (Rieke et al. 1980; Rieke 1988, and references therein), although there is still a considerable controversy as to whether the IMF is or can be universal (Scalo 1986; Gilmore and Roberts 1988). There may be some theoretical justification for this type of bias in starbursts (Larson 1986a, $b, 1987 a, 1988)$. Silk (1987c, 1988a) has argued that the high-mass star formation mode should be prevalent in starbursts at any redshift, and in protogalaxies in particular.

As a rule, supernovae contribute less than a percent of the total energy released in these starbursts. However, they must play a dominant role in the chemical enrichment of the gas. The first generation of massive stars provides the metals for the dust which will hide the subsequent generations. If the lowredshift starburst dwarf galaxies can be used as a guide, this process may take as little as $\sim 10^{7} \mathrm{yr}$ (Lequeux et al. 1981; Meier and Terlevich 1981; Hartmann et al. 1988). The formation of the $\mathrm{H}_{2}$ molecules and dust grains may be enhanced by the compression and radiative cooling in and downstream of the shocks in colliding protogalactic clouds (Shapiro and Kang 1987). Similar processes may be also responsible for the "dusting" of low-redshift IRAS galaxies.

\section{THE SMB MODELS}

We construct the SMB model spectra as follows. We represent the time history of the luminosity generated per comoving $\mathrm{Mpc}^{3}, G\left(t ; \sigma, t_{c}\right)$ as a Gaussian in the rest frame, specified by the dispersion $\sigma^{2}$, and the peak epoch $t_{c}$, corresponding to the redshift $z_{c}$. As a convenient number, the peak comoving bolometric luminosity density of $10^{11} L_{\odot} \mathrm{Mpc}^{-3}$ is used for the computation. The choice of a Gaussian history function is arbitrary, but we believe that other plausible shapes would produce very similar results. We are interested in the characteristic duration of the epoch of galaxy formation, in this case the full width at half-maximum, FWHM $\simeq 2.355 \sigma$. The spectrum of emitted radiation is assumed to be invariant, given 
either as $v B_{v}(45 \mathrm{~K})$, or as $v^{2} B_{v}(55 \mathrm{~K})$. The integration is done in redshift steps of $\Delta z=0.01$, from $z_{\min }$ corresponding to the epoch $t_{c}+3 \sigma$ (typically $z_{\min } \sim 6-8$ ), and up to $z_{\max }=20$; the history function is truncated outside these limits. At each redshift shell, we compute the volume element, $\Delta V$, corresponding to the observed solid angle of $1 \operatorname{arcsec}^{2}$, according to the formula (see, e.g., Condon 1988):

$$
\Delta V=\frac{4 \pi D^{2} c \Delta z}{\omega H_{0}(1+z)\left(1+\Omega_{0} z\right)^{1 / 2}},
$$

where $z$ is the central redshift of the shell, $\omega=5.3464 \times 10^{11}$ is the number of square arcseconds on the sphere, and $D$ is the distance given by Mattig's (1958) formula:

$$
D=\left(\frac{2 c}{H_{0}}\right) \frac{\Omega_{0} z+\left(\Omega_{0}-2\right)\left[\left(1+\Omega_{0} z\right)^{1 / 2}-1\right]}{\Omega_{0}^{2}(1+z)} .
$$

The redshift of the shell, $z$, is converted into the comoving time in the $\Omega_{0}=1$ case simply by:

$$
t(z)=\frac{2 t_{H}}{3(1+z)^{3 / 2}}
$$

and in a general $\Omega_{0}<1$ case as:

$$
\begin{aligned}
t(z)= & t_{H}\left[\frac{\sqrt{\Omega_{0} z+1}}{\left(1-\Omega_{0}\right)(1+z)}\right. \\
& \left.+\frac{\Omega_{0}}{2\left(1-\Omega_{0}\right)^{-3 / 2}} \ln \left(\frac{\sqrt{\Omega_{0} z+1}-\sqrt{1-\Omega_{0}}}{\sqrt{\Omega_{0} z+1}+\sqrt{1-\Omega_{0}}}\right)\right] .
\end{aligned}
$$

This is used in order to obtain the value of the history function $G\left(t ; \sigma, t_{c}\right)=G(z)$. The luminosity in a given redshift shell is then

$$
\Delta L\left(v_{*}, z\right)=v_{*}^{n} B_{v *}\left(T_{n}\right) G(z) \Delta V,
$$

where $v_{*}=v(1+z)$ is the rest frame frequency, and $v$ is the frequency in the observed frame. The corresponding flux con- tribution is then

$$
\Delta I_{v}(z)=\frac{L\left(v_{*}, z\right)}{4 \pi(1+z) D^{2}} .
$$

The redshifted flux contributions from successive redshift shells, $\Delta I_{v}(z)$, are then added up. Effectively, the total observed flux at a given frequency is given by

$$
I_{v}=\int_{z_{\min }}^{z_{\max }} \frac{v_{*}^{n} B_{v *}\left(T_{n}\right) G(z)}{4 \pi(1+z) D^{2}} \frac{d V}{d z} d z .
$$

This fixes the shape of the predicted spectrum, with the arbitrary normalization corresponding to the comoving peak bolometric luminosity density of $10^{11} L_{\odot} \mathrm{Mpc}^{-3}$. The true normalization factor is then determined by fitting the final spectrum to the observed SMB data. Growth of a typical model spectrum, indicating contributions from different redshift intervals, is illustrated in Figure 3.

A grid of models is computed for a range of $\sigma$ and $z_{c}$, for each of the four possible combinations of the template dust spectra and $\Omega_{0}$. The nominal values of $\chi^{2}$, derived from the first three data points, are used to evaluate the quality of the fit; the quantity optimized for each model is the renormalization factor of the peak luminosity density. We also require that the upper limit given by the fourth data point is not violated. For each value of $\sigma$, we then select the best $z_{c}$. There are three data points and one upper limit, and one or two fitting parameters, depending on whether one counts both $\sigma$ and $z_{c}$, or only $\sigma$, and thus there are one or two degrees of freedom, depending on one's statistical taste. An acceptable fit should have $\chi^{2} \leq 1-2$. It should also be emphasized that the error bars are dominated by the systematics, so that the values of $\chi^{2}$ do not have the meaning usually associated with a Gaussian statistic; nevertheless, they are an indicator of the quality of the fits.

The results are summarized in Figure 4. There is a notable parameter coupling in the models, in that the larger values of $\sigma$ require larger values of $z_{c}$. We search for the longest epoch of
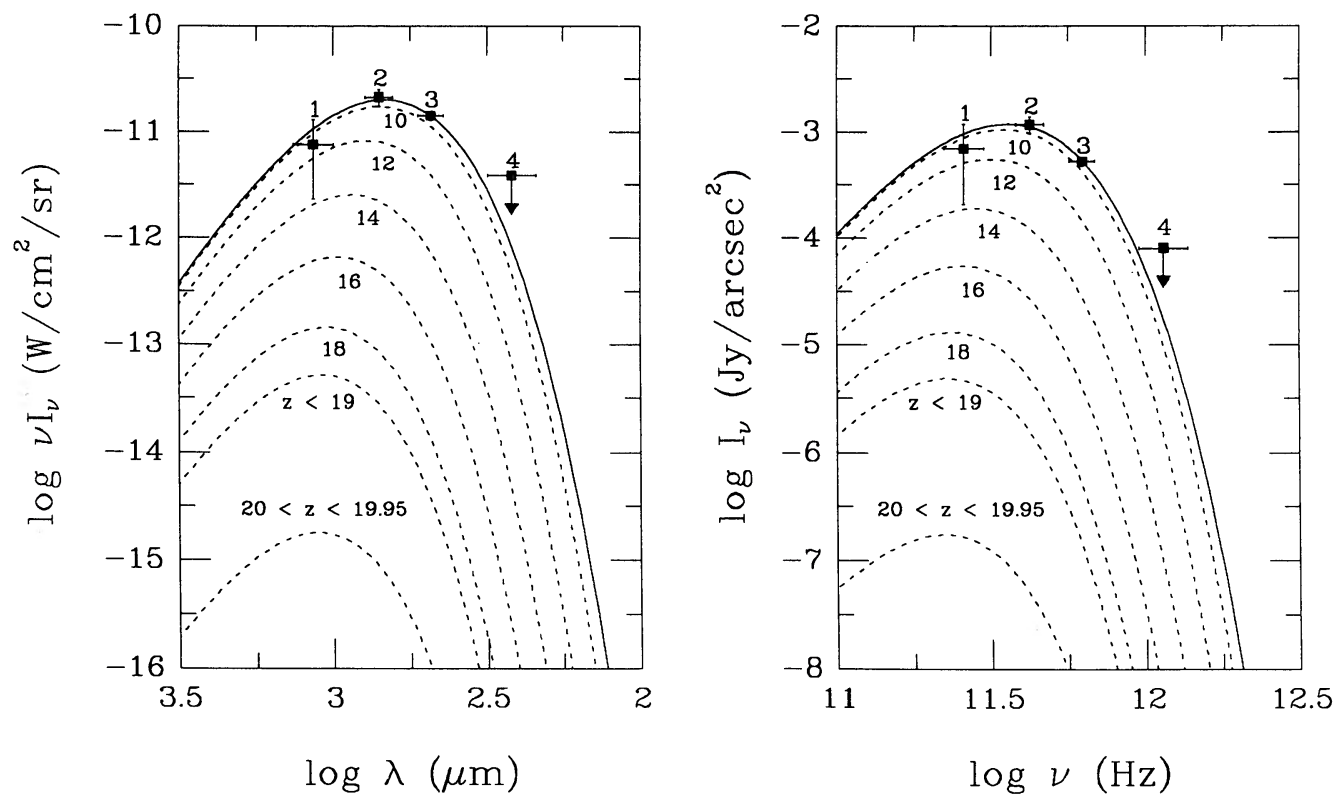

Fig. 3. - Contributions from different redshift intervals to a model spectrum of the SMB, assuming $n=2, \Omega_{0}=0.1$, and $\sigma=100$ Myr. Final spectrum is shown with the solid lines. Matsumoto et al.(1988) data points are shown as solid squares. The principal contribution to the total energy arises from $z \sim 10-13$. 


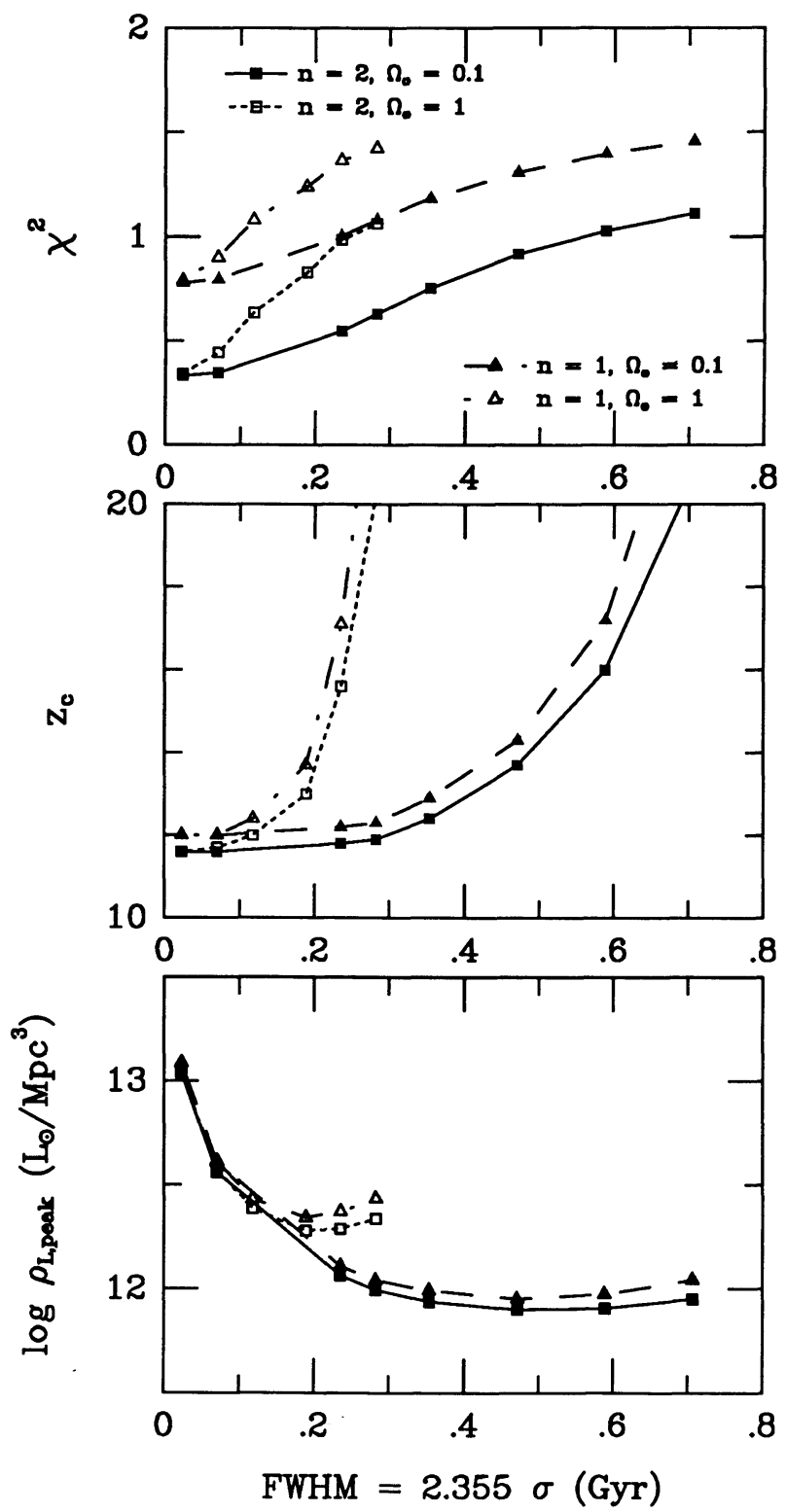

FIG. 4.-Summary of the results for the model parameters, plotted as functions of the FWHM of the luminosity history, $G\left(t ; z_{c}, \sigma\right)$. Values of $\Omega_{0}$ and $n$ are coded with different lines and symbols, as indicated. Top: the $\chi^{2}$ for the fits. Middle: dependence of the best-fit $z_{c}$ on the $\sigma$ (alias FWHM); note the coupling of the parameters. Bottom: normalization of the $G\left(t_{c}\right)$ for the best-fit $z_{c}$; for most models, it corresponds roughly to one Arp 220 per $\mathrm{Mpc}^{3} . H_{0}=75 \mathrm{~km}$ $\mathrm{s}^{-1} \mathrm{Mpc}^{-1}$ was assumed throughout.

galaxy formation (parameterized through $\sigma$ ) which is consistent with the data. The best fits are for the lowest values of $\sigma$. However, for the low-density models $\left(\Omega_{0}=0.1\right)$, values of $\sigma$ of up to $\sim(1-2.5) \times 10^{8} \mathrm{yr}$ are compatible with the data, corresponding to the FWHM $\sim 0.2-0.6$ Gyr. For the high-density models $\left(\Omega_{0}=1\right)$, the allowed widths are about a factor of 2 lower. These widths are constrained primarily by the observed shape of the SMB spectrum, which is fairly narrow itself. The widths of the model spectra are convolutions of the intrinsic spectra of our building blocks and the redshift width of the bursts. Larger durations of the bursts (redshift widths) and/or intrinsically broader spectra of the sources would violate the observed SMB spectrum shape. This is a potential difficulty common to all dust reradiation models of the SMB. Here we provide quantitative limits within the framework of our heuristic model. Some of the model spectra with the largest allowable widths of the epoch of galaxy formation are shown in Figure 5.

From the normalized luminosity histories, we can derive the total stellar mass processed in the burst, if we assume a value of the IMF-dependent parameter $\Xi$. The values of the mass yield, divided by the $\Xi$, are shown in Figure 6 . We require that $\Omega_{*} / \Xi \leq 0.02$, or if $\Omega_{*} \simeq 0.1, \Xi \leq 5$. Smaller values of $\Xi$ would allow smaller $\Omega_{*}$. As we demonstrated in $\S$ III, this is equivalent to the requirement that the IMF is biased toward massive stars, which may be the natural mode of star formation in starbursts.

\section{ADDITIONAL CONSTRAINTS AND CONSEQUENCES}

For a power-law IMF with the slope $-(1+x)$, the lockup fraction of the mass, $f_{\text {lock }}$, is given approximately as

$$
f_{\text {lock }}=\frac{M_{\mathrm{TO}}^{1-x}-M_{\mathrm{U}}^{1-x}}{M_{\mathrm{L}}^{1-x}-M_{\mathrm{U}}^{1-x}},
$$

where $M_{\mathrm{TO}}$ is the main sequence turnoff mass at that time. For our models, the relevant look-back time is $\sim 10 \mathrm{Gyr}$, and $M_{\text {To }} \sim M_{\odot}$. For the range of IMF parameters required above, we obtain $f_{\text {lock }}>0.35$, and typically $f_{\text {lock }} \sim 0.9$. Thus, a large portion of the mass processed in the starbursts, and thus $\Omega_{*}$, should be now locked in low-luminosity and dark stellar remnants, i.e., cool white dwarfs, neutron stars, or stellar-mass black holes.

Since the $M / L$ ratios in the central parts of bulges and ellipticals are not greater than $\sim 10$, this population would have to be distributed in a less concentrated manner than the visible light today. An additional constraint on the distribution of neutron stars and stellar black holes in this population may be obtainable from the requirement that the number of accreting binaries in the Galactic bulge does not exceed the observed numbers. For the values of $\Omega_{*}$ greater than about a couple of percent, a large fraction of this processed material may have to be associated with groups and clusters, rather than galaxies. This could happen, for example, if the starbursts occur in smaller protogalactic units, some of which can be tidally disrupted. This distribution requirement is possibly the most important problem for all models of baryonic dark matter, including ours.

In order to reprocess the radiation from young stars into the infrared, some minimal amount of dust is required. Estimates of this quantity are fraught with difficulties (see Hildebrand 1983), but Soifer et al. (1987) provide the following useful formula:

$$
M_{\text {dust }} \simeq 10^{-4}\left(\frac{L_{\text {bol }}}{L_{\odot}}\right)\left(\frac{T_{\text {dust }}}{40 \mathrm{~K}}\right)^{-5} M_{\odot} .
$$

The peak luminosity densities can thus be converted to the comoving dust densities in the midstarburst. The latter cannot exceed the amount of metals generated by that time, $\Omega_{*} Z$. If we associate the stellar populations generated in these bursts with the present-day metal-rich old stellar populations, their final metallicities are in the range $Z \sim(1-2) Z_{\odot}$, where $Z_{\odot}=$ 0.018 . Thus, the plausible midburst metallicities are $Z \sim 0.01$. With $\Omega_{*} \sim 0.1$, the dust densities required by our models are well within the allowed range, as illustrated in Figure 6. Detailed modeling of the chemical enrichment with recycling of the processed material, the lockup of some fraction of it in 

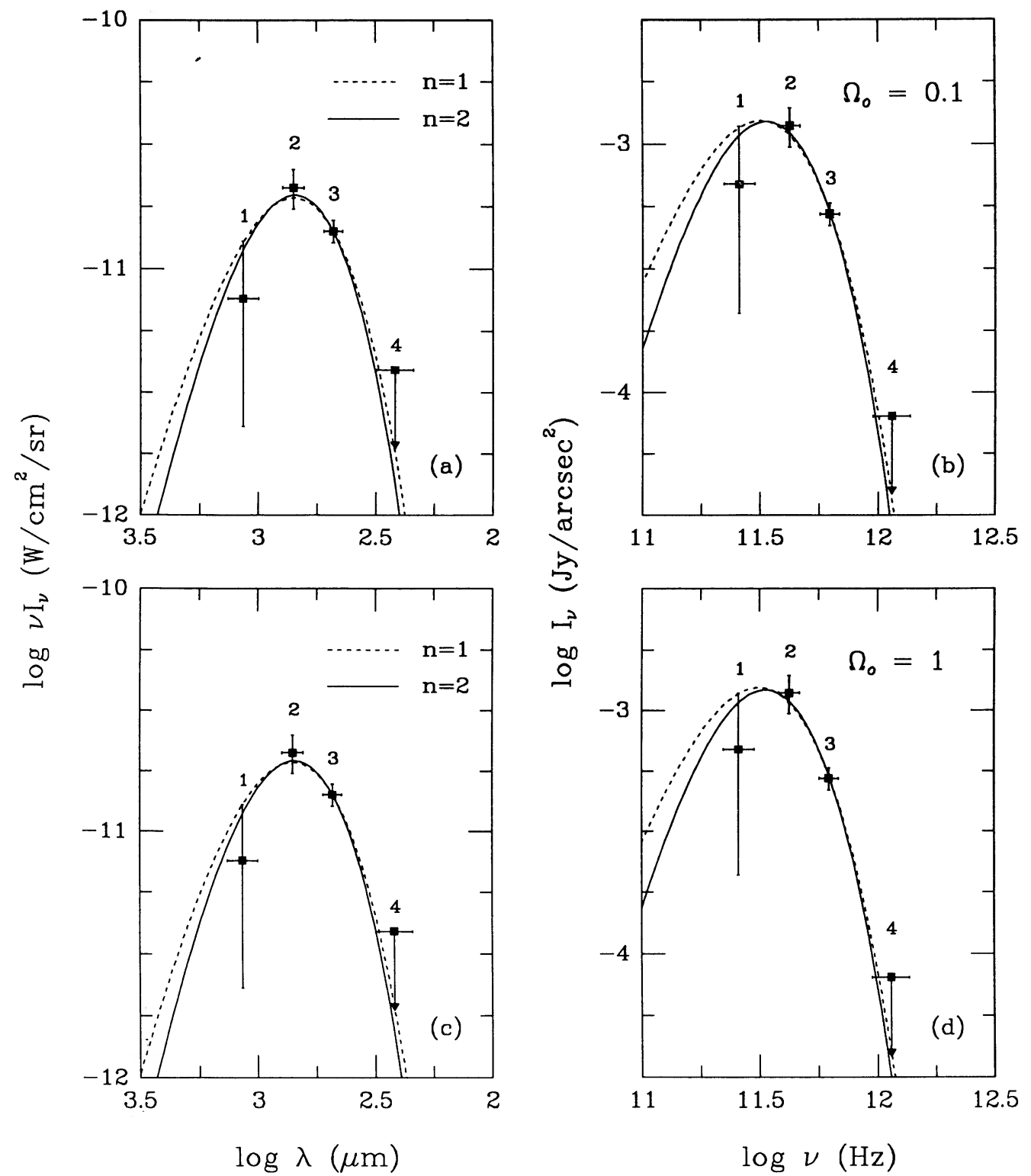

Fig. 5.-Representative SMB spectra of models with some of the largest allowed values of $\sigma: 200 \mathrm{Myr}$ for $\Omega_{0}=0.1([a]$ and $[b])$, and $100 \mathrm{Myr}$ for $\Omega_{0}=1([c]$ and [d]). The $n=2$ models are shown with solid lines, and the $n=1$ models are shown with the dotted lines.

stellar remnants, and its dependence on the IMF parameters, is outside the scope of this paper. A future investigation in this direction could provide an important consistency check for our scenario.

Finally, we can estimate the expected surface density of SMB sources from the following simple argument. Let us represent the projected shell of galaxy formation as a uniform distribution of sources with luminosities $L_{\mathrm{SMB}}=f_{11} \times 10^{11} L_{\odot}$, at the constant $z_{c}=12$. For the $\Omega_{0}=0.1$ models, the surface density of sources required to reproduce the observed brightness of the SMB is $85 / f_{11} \operatorname{arcsec}^{-2}=1.1 \times 10^{9} / f_{11} \mathrm{deg}^{-2}$, corresponding to the rms of $0.11 \sqrt{ }\left(f_{11}\right)$ arcsec between the sources. For the $\Omega_{0}=1$ models, the surface density of sources is $9.4 / f_{11}$ $\operatorname{arcsec}^{-2}=1.2 \times 10^{8} / f_{11} \mathrm{deg}^{-2}$, corresponding to the rms of $0.33 \sqrt{ }\left(f_{11}\right)$ arcsec between the sources. The SMB should thus be rather smooth on scales greater than a few arcseconds. We note that the sources of the SMB need not represent progeni- tors of individual present-day galaxies. They are more likely to be protogalactic fragments, probably participating in an extensive merging at later epochs. Thus, galaxy counts at lower redshifts are unlikely to provide strong constraints on this prediction. Also, the presence of any incipient large-scale structure would increase the fluctuations relative to our purely Poissonian estimates.

An unknown geometrical factor in our models is the dust covering fraction, and we have tacitly assumed that all starbursts are completely shrouded. It is reasonable to expect some patchiness of the dust, and a fraction of the primary starlight could escape unobscured. This raises an interesting possibility of detecting such radiation. Indeed, in another rocket-borne experiment, Matsumoto, Akiba, and Murakami (1987, 1988) measured the intensity of the diffuse near-infrared radiation in the wavelength range $\lambda \sim 1.1-5.4 \mu \mathrm{m}$. They claim that there is an isotropic component detected at $\lambda \sim 2-4 \mu \mathrm{m}$ which cannot 


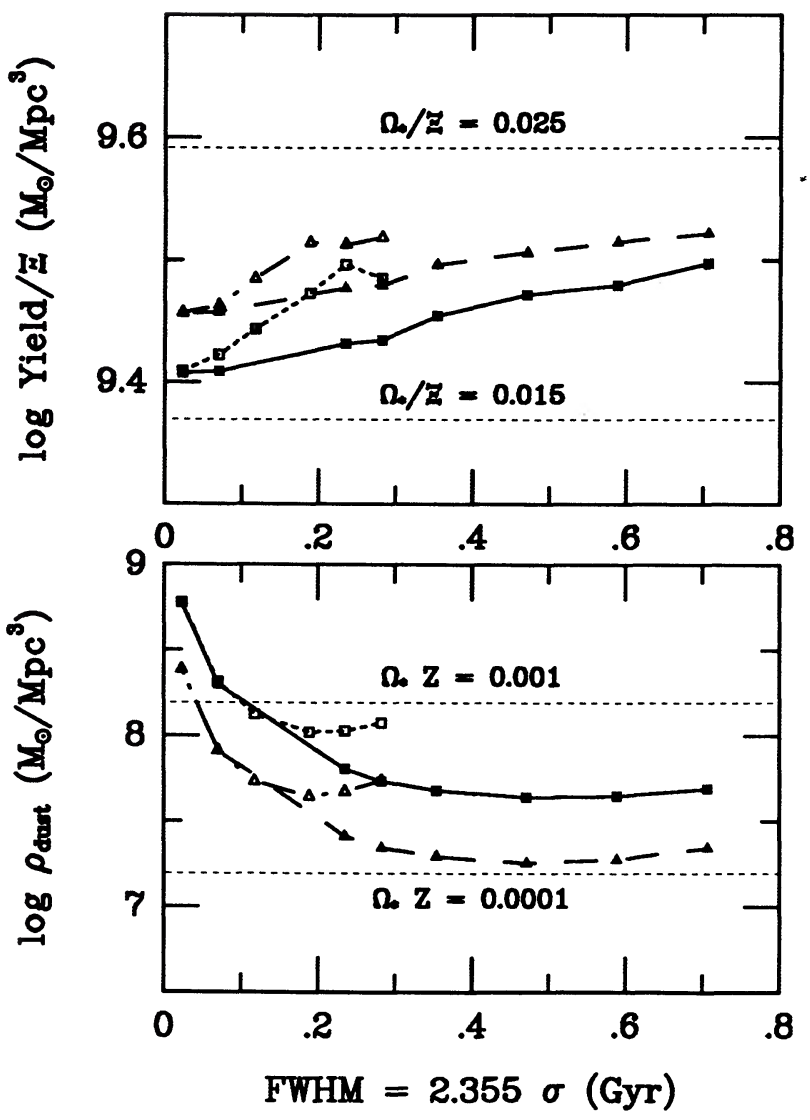

FIG. 6.-Top: mass production in the best-fit models, renormalized by the parameter $\Xi$. Horizontal lines indicate the limits from the resulting cosmological density of stars generated in the bursts, $\Omega_{*}$. If $\Omega_{*}=0.1$, the maximum allowed $\Xi$ is $\sim 5$. For lower $\Omega_{*}$, lower values of $\Xi$ are required. Bottom: the required dust mass density, determined from the peak luminosity densities for the best-fit models. Horizontal lines indicate the amounts of metals generated by the time $t=t_{c}$, expressed as contribution to $\Omega_{*}$, and assuming that all metals can be used to form the dust. Different values of $\Omega_{0}$ and $n$ are coded with different lines and symbols, as in Fig. 4.

be easily attributed to any known astronomical cause or instrumental effect. They find that in the $K(2.2 \mu \mathrm{m})$ band, $v I_{v}=(1.0 \pm 0.4) \times 10^{-11} \mathrm{~W} \mathrm{~cm}^{-2} \mathrm{sr}^{-1}$, and in the $L(3.8 \mu \mathrm{m})$ band, $v I_{v}=(1.1 \pm 0.4) \times 10^{-11} \mathrm{~W} \mathrm{~cm}^{-2} \mathrm{sr}^{-1}$; the $K$ band measurement is deemed more reliable, The corresponding surface brightness in the $K$ band is $I_{v}=1.8 \pm 0.6 \mu \mathrm{Jy} \mathrm{arcsec}^{-2}$, or $\mu_{K}=21.4 \pm 0.4 \mathrm{mag} \operatorname{arcsec}^{-2}$. Thus, the energy density in this near-infrared background (if it indeed exists) is about $10 \%$ of that in the SMB. It is tempting to speculate that this background may represent leakage of the starlight from our hypothetic starbursts at $z \sim 10-15$, viz., Ly $\alpha$ emission and the Lyman continuum. Assuming our estimates of the number of sources per square arcsecond, we conclude that the individual sources may be as faint as $K \sim 24$. Anisotropy of the extragalactic background light in the $K$ band on scales of $\sim 10^{\prime \prime}-30^{\prime \prime}$ and $\sim 60^{\prime \prime}-300^{\prime \prime}$ was measured by Boughn, Saulson, and Uson (1986). Extrapolation of their limits to the $K \sim 24$ level suggests that there should be at least $1-10$ sources $\operatorname{arcsec}^{-2}$, in a good agreement with our estimates for the surface density of the SMB sources. The limits are actually softer, because they assume that the source sizes would be smaller that the rms between the sources, which is unlikely to be the case here $(0.1$ corresponds to $0.65 h_{75}^{-1} \mathrm{kpc}$ at $z=12$ for $\Omega_{0}=0.1$, or to $0.22 h_{75}^{-1} \mathrm{kpc}$ for $\Omega_{0}=1$ ).

\section{A POSSIBLE CONNECTION WITH THE X-RAY BACKGROUND}

A population of starburst galaxies at large redshifts which can produce the SMB could also be an important contributor to the diffuse extragalactic soft X-ray background (XRB). The origin of the XRB is still not completely understood; good recent reviews include, e.g., those by Setti and Woltjer (1982), Giacconi and Zamorani (1987), or Hamilton and Helfand (1987). The energy density of the XRB is $u_{\text {XRB }} \sim 2 \times 10^{-17}$ ergs $\mathrm{cm}^{-3}$ in the Einstein 1-3 keV band (Giacconi et al. 1979), and a comparable density at lower energies (see also Daly and Turner 1988). Most likely, quasars and active galactic nuclei (AGNs) can account for a large fraction of the background (Schmidt and Green 1986; Schwartz and Tucker 1988, and references therein). They can contribute at least $\sim 30 \%$ of the background at $2 \mathrm{keV}$, but probably not much more unless some very special allowances are made for their evolution. The isotropy and spatial fluctuations of the XRB require a high surface density of sources, greater than several thousand per square degree (Hamilton and Helfand 1987; Barcons and Fabian 1989). No known population of objects at low redshift fulfills all the requirements imposed by the data. Starburst galaxies or protogalaxies at large redshifts have been proposed as a possible major contributor to the XRB (Bookbinder et al. 1980; Weedman 1987; Daly 1987; Fabbiano 1988; Griffiths 1989).

X-ray emission has been observed from star-forming regions and interacting galaxies (Weedman et al. 1981; Fabbiano and Trinchieri 1983, 1984; Fabian 1985; Fabbiano 1989, and references therein). Prominent X-ray emission has been observed from both of our template galaxies, M82 (Watson, Stanger, and Griffiths 1984; Fabbiano 1988; Schaaf et al. 1989), and Arp 220 (Eales and Arnaud 1988). About equal fractions of the $\mathrm{X}$-ray luminosity in these systems come from the compact sources, probably accreting binaries or buried supernova remnants, and a diffuse component distributed on the scale of several kiloparsecs. The origin of the diffuse $\mathrm{X}$-ray emission is unclear, but one possible mechanism may be the inverse Compton scattering of the infrared photons generated in the starburst on the hot plasma from the merger shocks and supernova remnants. The spectra are soft, corresponding to the bremsstrahlung temperatures of $\sim 10 \mathrm{keV}$.

The ratios of $\mathrm{X}$-ray and infrared luminosities in these objects are $L_{\mathrm{X}} / L_{\mathrm{IR}} \sim(1-5) \times 10^{-4}$. It may be more than a coincidence that the ratio of the energy density in the soft XRB, a few times $10^{-17}$ ergs $\mathrm{cm}^{-3}$, and that of the SMB, $\sim 10^{-13} \mathrm{ergs} \mathrm{cm}^{-3}$, is of the similar order. This situation would arise naturally if the sources of the SMB give rise to at least a part of the XRB. Our estimated surface density of the SMB sources also easily satisfies the lower limit on the surface density of the XRB sources by Hamilton and Helfand (1987) and Barcons and Fabian (1989).

The X-ray spectra of the starbursts are probably too soft to account for a substantial fraction of the background at higher energies, $>10 \mathrm{keV}$, say, but that is a common affliction of most models for the origin of the XRB. It is possible that there is a substantial XRB at energies $\leq 0.1 \mathrm{keV}$, which are still poorly explored. An old measurement by Palmieri et al. (1971) detected $u_{\mathrm{XRB}} \sim(4-11) \times 10^{-17} \mathrm{ergs} \mathrm{cm}^{-3} \mathrm{keV}^{-1}$ at $0.25 \mathrm{keV}$, but an unknown fraction of this flux could be of a Galactic origin; for an up-to-date discussion, see Burrows (1989) and 
references therein. It would be important to obtain better measurement of the extragalactic component of the soft XRB at energies $<0.5 \mathrm{keV}$.

\section{DISCUSSION}

To summarize, we can produce the SMB in dust-shrouded starbursts at typical redshifts $z_{c} \sim 12-15$, and durations $\sim(1-$ 5) $\times 10^{8} \mathrm{yr}$. The final product of this epoch of vigorous star formation may be the present-day ellipticals, bulges, and possibly also old stellar disks, and at least a part of the massive halos. Metal-poor old stars would be the stars formed in the early phases of the bursts, but the main product would be the metal-rich old stellar population. A large fraction of the baryonic density of the universe, $\Omega_{b}$, would be locked in the stars and stellar remnants of this population, i.e., $\Omega_{*} \sim 0.1$, depending strongly on the IMF; in general, we require that the IMF was biased toward the high-mass stars. This mass, consisting mainly of low-luminosity and dark stellar remnants, would have to be distributed in a manner less concentrated than the visible stars. In other words, it would constitute at least a large fraction of galactic dark halos. Similar models have been proposed, e.g., by Larson Silk (1987a), Silk (1988b), Silk and Tamanaha (1989) and Silk (1989, private communication.) Luminous, dusty starburst protogalaxies may also be responsible for a substantial fraction of the soft XRB and of the near-infrared background, which may have been detected by Matsumoto, Akiba, and Murakami $(1987,1988)$.

The discovery of primeval galaxies (galaxies undergoing their initial collapse, or their first major burst of star formation at large redshifts) is one of the central goals of modern observational cosmology. The early searches were reviewed by Davis (1980) and Koo (1986). It is intriguing that some objects have been found recently which can be interpreted as being young or forming galaxies at the moderately high redshifts, $z \sim 1.8$ 3.8 (Djorgovski et al. 1985, 1987; McCarthy et al. 1988; Spinrad 1987; Djorgovski 1988b; Cowie and Lilly 1989; Chambers, Miley, and van Breugel 1989; McCarthy et al. 1990). On the other hand, the results on red field galaxies at $z \leq 0.6-0.8$ by Hamilton (1985), on rich clusters at $z \leq 0.92$ (Gunn, Hoessel, and Oke 1986; Oke 1988), and the $z=3.4$ radio galaxy found by Lilly (1988), suggest that at least some fraction of these systems formed at $z>5$. Radio galaxies are now known out to $z \simeq 3.8$ (Chambers, Miley, and van Breugel 1989), and quasars are known out to $z \simeq 4.7$, without any evidence for a sharp cutoff (Schmidt, Gunn, and Schnieder 1989). Faint field galaxy counts by Tyson $(1988 a, b)$ indicate that there was no narrow burst of unobscured galaxy formation at $z<6$. If there ever was a well-defined epoch of galaxy formation, it must have occurred at larger redshifts, and/or the protogalaxies were shrouded in dust.

We next address the question of timing, both of the epoch of initial galaxy formation and its width. The redshifts of the peak star-forming activity in our models $\left(z_{c} \sim 12-15\right)$ correspond to the look-back times of $\sim 11.5 h_{75}^{-1} \mathrm{Gyr}$ for $\Omega_{0}=0.1$, or $\sim 8.5 h_{75}^{-1}$ Gyr for $\Omega_{0}=1$. The age dating of the Galactic globular clusters yields the values in the range 12-18 Gyr (Zinn 1986; VandenBerg 1988; Sarajedini and King 1988); the age dating of the Galactic bulge and nearby elliptical galaxies is much less certain but is consistent with this range (Renzini 1986; O'Connell 1986, 1988; Frogel 1988). The age of the Galactic disk in the solar neighborhood has been estimated to $\sim 9 \pm 2$ Gyr from the luminosity function of white dwarfs (Winget et al. 1987). Nuclear cosmochronometers give similar values for the age of our Galaxy, $\sim 11 \pm 2$ Gyr (Fowler 1987). The estimated ages of our galaxy and old stellar systems are thus in a good agreement with the look-back times to the initial burst of galaxy formation in our models.

The duration of the epoch of the initial galaxy formation is also of some interest. Recall that the free-fall time of a selfgravitating cloud without dissipation is given approximately as

$$
t_{\mathrm{ff}} \simeq 1.7 \times 10^{6} \mathrm{yr}\left(\frac{M}{10^{11} M_{\odot}}\right)^{-1 / 2}\left(\frac{R_{\text {init }}}{\mathrm{kpc}}\right)^{3 / 2}
$$

where $M$ is the mass of the cloud, and $R_{\text {init }}$ is its initial radius at the redshift of collapse. For a reasonable range of $M$ and $R_{\text {init }}$, $t_{\mathrm{ff}} \sim 10^{8} \mathrm{yr}$. Presence of dissipation could accelerate the cloud collapse. Thus, the allowed widths of the epoch of galaxy formation from our models are about equal to the expected freefall times, or slightly larger. The formation on a time scale $\sim t_{\mathrm{ff}}$ is in agreement with the classical picture by Eggen, LyndenBell, and Sandage (1962), and probably most of the more modern data on the old stellar populations in our Galaxy (Sandage and Fouts 1987; Sandage 1986, 1987, and references therein).

The data on the ages of old stellar systems at $z \sim 0-1$, and the existence of active galaxies at $z>3-4$, are thus fully consistent with an initial epoch of galaxy formation at $z>10$. It is also very likely that a substantial, partly dissipative, or dissipationless merging activity happened at a range of redshifts, following the initial burst, and there is some observational evidence for such processes (Silk and Norman 1981; Silk $1987 a, b$; Baron and White 1987; Silk and Szalay 1987; Djorgovski $1988 a, b$ ). In particular, most elliptical galaxies may have grown by a post-initial formation merging. This "gradual galaxy formation" picture does not preclude a possibility that there was an initial, narrow burst of highly dissipative galaxy formation at $z \geq 10$.

The cold dark matter (CDM) scenarios and $N$-body models of large-scale structure and galaxy formation predict a vigorous merging activity at intermediate redshifts, $z \sim 1-3$, which is often interpreted as the epoch of galaxy formation in this context (Frenk et al. 1988; Silk 1987a, b; Baron and White 1987; Silk and Szalay 1988). This is really a plausibility argument: the CDM models deal with the gravitational processes, viz., clustering and merging of dark halos, and star formation is added to them in an ad hoc fashion, typically through some arbitrary biasing scheme. Even if the CDM scenario is basically correct and describes correctly the evolution of dark halos and the large-scale structure, it does not yet contain the physics of star formation in protogalaxies. It does not predict unambiguously or depend strongly on the redshift at which the first, or the most, old stars form. The epochs of the maximum star formation and of the maximum merger rate need not coincide.

Since the universe appears to be fairly transparent out to $z \sim 3-5$ (Fall and Pei 1989; but see also Ostriker and Heisler 1984 , or Heisler and Ostriker 1988), the dust from the initial starbursts has to be somehow evaporated or consumed by the subsequent star formation. The processes which operate in low-redshift ELFs may be conducive to the formation of active galactic nuclei (Sànders et al. 1988a, b; Scoville 1988; Norman and Scoville 1988). The initial burst of galaxy formation at $z \sim 8-15$ could have been naturally followed by the appearance of quasars at $z \sim 4-8$; their ultraviolet and X-ray (UVX) 
radiation could help to reionize the ISM of the host galaxies as well as the IGM.

\section{MODEL PREDICTIONS AND FUTURE TESTS}

Repeated and improved measurements of the spectrum and intensity of the SMB would provide the most important constraints for the quantitative aspects of our model. Another rocket experiment is being prepared by the Berkeley-Nagoya group (Lange 1989, private communication). The FIRAS experiment on the $C O B E$ satellite (Mather 1982) could provide a definitive measurement within this year. Improved measurements of the shape of the SMB spectrum provides the constraints on the width of the epoch of galaxy formation.

The two strongest predictions of our model are: (1) the SMB should be extraordinarly smooth, unless there is some fundamental error in our understanding of the large-scale structure or cosmological models; (2) most of the dark matter within the visible parts of galaxies should consist of faint or dark stellar remnants (e.g., cool white dwarfs or neutron stars).

If there are $\sim 10$ or more SMB sources per $\operatorname{arcsec}^{2}$, the relative fluctuations in a $\sim 10^{\prime \prime}$ beam should not exceed $1 \%$. This may be testable in the submillimeter region with the present generation of instruments, or in the near future. Kreysa and Chini (1989) limits at $1.3 \mathrm{~mm}$ with the beam size of $\sim 11^{\prime \prime}$ and on the scales $\sim 30^{\prime \prime}$ are interestingly close this estimate, although the SMB intensity at that wavelength is already $\sim 10 \%$ of its peak value. Kreysa and Chini limits of $\delta S_{\mathrm{rms}} \simeq$ $0.77 \mathrm{mJy}$ beam $^{-1}$ translate to fluctuations of $\Delta I_{v} / I_{v} \leq 1 \%$ per $100 \operatorname{arcsec}^{2}$ beam, or to the Poissonian fluctuations corresponding to $\geq 100$ sources per $\operatorname{arcsec}^{2}$. This experiment is well worth redoing in an independent way or at a deeper level.

If the $K$ band background claimed by Matsumoto, Akiba, and Murakami $(1987,1988)$ is generated by the same population of protogalaxies, it may be detectable with the modern infrared imagers and $10 \mathrm{~m}$ class telescopes now under construction, or the second-generation Hubble Space Telescope instruments, either via direct imaging, or statistically, though the analysis of intensity fluctuations on the sky. If the same sources are responsible for the soft X-ray background, similar measurements could be possible with the $A X A F$ satellite.

It may also be possible to find a spectroscopic signature of the starbursts at $z \sim 10-15$. Many of the commonly observed lines from the low-redshift dusty starbursts would be redshifted into the region which is difficult to observe from the ground, $\lambda \sim 10-100 \mu \mathrm{m}$ : the recombination lines of hydrogen, e.g., $\mathrm{Pa} \alpha$ $1.875 \mu \mathrm{m}, \mathrm{Br} \alpha 4.05 \mu \mathrm{m}$, or $\mathrm{Br} \gamma 4.05 \mu \mathrm{m}$, the $\mathrm{H}_{2}$ molecular lines at 2.22 and $2.12 \mu \mathrm{m}$, or the polycyclic aromatic hydrocarbon (PAH) band at $3.3 \mu \mathrm{m}$. Their luminosities are typically $\sim 1 \%$ of the total bolometric flux, which would make them just barely detectable with the SIRTF satellite, at its sensitivity limit of $\sim 10 \mu \mathrm{Jy}$ at these wavelengths (Werner and Eisenhardt 1988). A number of the fine structure lines of metals, e.g., [O III] 88.36 $\mu \mathrm{m}$, [O I] $145.53 \mu \mathrm{m}$, or [C II] $157.74 \mu \mathrm{m}$, would be redshifted into the atmospheric windows at millimeter wavelengths and may be detectable with the next generation of receivers. A spectroscopic signature of the leaked Ly $\alpha$ emission and Lyman continuum may be detectable with deep near-infrared area spectroscopy, e.g., Fabry-Perot imaging.

Rowan-Robinson (1986) analyzed the IRAS background at $100 \mu \mathrm{m}$ and concluded that there is a residual component which cannot be accounted for by the infrared cirrus, or other Galactic sources, on the level $I_{v}=120-150 \mu \mathrm{Jy} \operatorname{arcsec}^{-2}$, or $v I_{v}=1.5-1.8 \times 10^{-11} \mathrm{~W} \mathrm{~cm}{ }^{-2} \mathrm{sr}^{-1}$, which is considerably higher than any thermal extrapolation from the SMB. It is possible that this emission, if real, comes from the redshifted $(z \sim 11-15)$ PAH bands at 6.2, 7.7, and $8.6 \mu \mathrm{m}$.

Our second major prediction is that substantial numbers of old, relic white dwarfs (RWD) should be found in the Galactic bulge and halo. Silk and Tamanaha (1989) discuss this possibility in a very similar context. In fact, this prediction is generic for almost any model in which a substantial fraction of the dark matter is in stellar remnants. We can make a rough estimate of the relevant parameters of such population as follows. The cooling time for white dwarfs is given by Shapiro and Teukolsky (1983):

$$
\tau_{c}=5.13 \times 10^{7} \mathrm{yr} A^{-1}\left(\frac{M}{M_{\odot}}\right)^{5 / 7}\left(\frac{L}{L_{\odot}}\right)^{-5 / 7},
$$

where $A \simeq 6$ is the mean atomic weight. Assuming $\tau_{c} \sim 10$ Gyr, and $M \sim 0.5 M_{\odot}$, we estimate the characteristic luminosity $L_{\text {RWD }} \sim 2.5 \times 10^{-5} L_{\odot}$, or $M_{\text {RWD }} \sim 16.2$. Bahcall (1986) estimates the local halo density to be $\rho_{\text {halo }} \simeq 0.009 M_{\odot} \mathrm{pc}^{-3}$. If about one-half of that is in the RWDs, there should be $\sim 4 \times 10^{4}$ of them within $100 \mathrm{pc}$. The corresponding surface density would be $\sim 1 \mathrm{deg}^{-2}$, at the magnitude level $m_{\mathrm{RWD}} \sim 21$. Going out to $1 \mathrm{kpc}$, the surface density would be $\sim 10^{3} \mathrm{deg}^{-2}$, at the magnitude level $m_{\mathrm{RWD}} \sim 26$. These numbers are just a rough guideline, but searches now planned or underway could detect such populations of old white dwarfs at high galactic latitudes. Their proper motions would be typical of the halo stars, $\sim 0.5-5$ arcsec $\mathrm{yr}^{-1}$. In addition, it may be possible to detect more luminous white dwarfs in Baade's Window and other low-extinction windows in the bulge, with the Hubble Space Telescope. Failure to detect old white dwarfs in the halo and bulge, in the numbers which would make them a dominant contributor to the baryonic mass of the Galaxy, would present a severe difficulty for our model.

We are indebted to many colleagues for stimulating and informative discussions, and in particular to Andrew Lange and Joseph Silk. We thank Nick Scoville and the referee for useful comments on the manuscript. S. D. acknowledges the stimulating atmosphere of the Aspen Center for Physics, and the workshop organized by Jim Truran, which motivated this study. This work was supported in part by the Alfred P. Sloan Foundation and California Institute of Technology (S. D.), and a National Science Foundation fellowship (N. W.). The motivation for this work was in part to prepare a scientific rationale for the proposed NASA Submillimeter Explorer mission. The publication of this paper was supported in part by the funds from NASA.

\section{REFERENCES}

Adams, F. C., Freese, K., Levin, J., and McDowell, J. C. 1989, Ap. J, 344, 24

Alcock, C., Fuller, G. M., and Mathews, G. J. 1987, Ap. J., 320, 439.

Applegate, J., and Hogan, C. J. 1985, Phys. Rev. D, 31, 3037.

Applegate, J., Hogan, C. J., and Scherrer, R. J. 1987, Phys. Rev. D, 35, 1151.

Bahcall, J. 1986, Ann. Rev. Astr. Ap., 24, 577.

Barcons, X., and Fabian, A. 1989 , M.N.R.A.S., 237, 119

Baron, E., and White, S. D. M. 1987, Ap. J., 322, 585.

Bartlett, J. G., and Silk, J. 1989, Berkeley CfPA preprint TH-89-006.
Bersanelli, M., Witebsky, C., Bensadoun, M., De Amici, G., Kogut, A., Levin, S., and Smoot, G. 1989, Ap. J., 339, 632 .

Binney, J. 1977, Ap. J., 215, 483.

Boesgaard, A. M., and Steigman, G. 1985, Ann. Rev. Astr. Ap., 23, 319.

Bond, J. R., Carr, B. J., and Hogan, C. J. 1986, Ap. J., 306, 428.

Bookbinder, J., Cowie, L. L., Krolik, J. H., Ostriker, J. P., and Rees, M. 1980, Ap. J., 237, 647 .

Boughn, S. P., Saulson, P. R., and Uson, J. M. 1986, Ap. J., 301, 17. 
Burrows, A. 1989, Ap. J., 340, 775.

Carr, B. J. 1987, in Comets to Cosmology, ed. A. Lawrence (Berlin: Springer) p. 256.

1989, in Epoch of Galaxy Formation, ed. C. Frenk, R. Ellis, T. Shanks,

A. Heavens, and J. Peacock (Dordrecht: Kluwer), p. 227

Chambers, K., Miley, G., and van Breugel, W. 1989, in preparation.

Condon, J. 1988, in Galactic and Extragalactic Radio Astronomy, ed. G. Bers-

chuur and K. Kellerman (Berlin: Springer), p. 641.

Cowie, L. L., and Lilly, S. J. 1989, Ap. J. (Letters), 336, L41.

Crane, P., Hegyi, D. J., Mandolesi, N., and Danks, A. C. 1986, Ap. J., 309, 822.

Daly, R. 1987, Ap.J., 322, 20.

. 1988, Ap.J.(Letters), 333, L1.

Daly, R., and Turner, E. L. 1988, Comments Ap., 12, 219.

Dar, A., Loeb, A., and Nussinov, S. 1989, Ap. J.(Letters), 338, L41.

Davis, M. 1980, in IAU Symposium 92, Objects at High Redshift, ed. G. O. Abell and P. J. E. Peebles (Dordrecht: Reidel), p. 57.

de Lapparent, V., Geller, M. J., and Huchra, J. P. 1989, Ap. J., 332, 44

Djorgovski, S. 1988a, in Starbursts and Galaxy Evolution, ed. T. X. Thaun, T.

Montmerle, and J. Tran Thanh Van (Gif sur Yvette: Editions Frontières) p. 401 .

. 1988b, in Towards Understanding Galaxies at Large Redshift, ed. R. G. Kron and A. Renzini (Dordrecht: Kluwer), p. 259.

Djorgovski, S., Spinrad, H., McCarthy, P., and Strauss, M. 1985, Ap. J (Letters), 299, L1.

Djorgovski, S., Strauss, M., Perley, R., Spinrad, H., and McCarthy, P. 1987, A.J., 93, 1318.

Draine, B. T., and Lee, H. M. 1984, Ap. J., 285, 89.

Draine, B. T., and Shapiro, P. R. 1989, Ap. J.(Letters), 344, L5.

Eales, S. A., and Arnaud, K. A. 1988, Ap. J., 324, 193.

Efstathiou, G., and Silk, J. 1983, Fund. Cosm. Phys., 9, 1.

Eggen, O., Lynden-Bell, E., and Sandage, A. 1962, Ap. J., 136, 748.

Emerson, J. P. 1988, in Formation and Evolution of Low Mass Stars, ed. A. K.

Dupree and M. T. V. T. Lago (Dordrecht: Kluwer), p. 21.

Emerson, J. P., Clegg, P. E., Gee, G., Cunningham, C. T., Griffin, M. J., Brown,

L. M. J., Robson, E. I., and Longmore, A. J. 1984, Nature, 311, 237.

Fabbiano, G. 1988, Ap. J., 330, 672.

1989, Ann. Rev. Astr. Ap., 27, 87

Fabbiano, G., and Trinchieri, G. 1983, Ap. J. (Letters), 266, L5.

. 1984, Ap. J., 286, 491.

Fabian, A. C. 1985, in Star-Forming Dwarf Galaxies and Related Objects, ed. D.

Kunth, T. X. Thaun, and J. Tran Thanh Van (Paris: Editions Frontieres) p. 383.

Fall, S. M., and Pei, Y. C. 1989, Ap. J., 337, 7.

Fowler, W. A. 1987, Quart. J.R.A.S., 28, 87.

Frenk, C. S., White, S. D. M., Davis, M., and Efstathiou, G. 1988, Ap. J., 327, 507.

Frogel, J. 1988, Ann. Rev. Astr. Ap., 26, 51

Giacconi, R., et al. 1979, Ap. J. (Letters), 234, L1.

Giacconi, R., and Zamorani, G. 1987, Ap. J., 313, 20

Gilmore, G., and Roberts, M.S. 1988, Comments Ap., 12, 123.

Griffiths, R. E. 1989, in Epoch of Galaxy Formation, ed. C. Frenk, R. Ellis, T.

Shanks, A. Heavens, and J. Peacock (Dordrecht: Kluwer), p. 235.

Gunn, J. E., Hoessel, J. G., and Oke, J. B. 1986, Ap. J., 306, 30

Halpern, M., Benford, R., Meyer, S., Muehlver, D., and Weiss, R. 1988, Ap. J., 332, 596 .

Hamilton, D. 1985, Ap.J., 297, 371.

Hamilton, T. T., and Helfand, D. J. 1987, Ap. J., 318, 93

Hartmann, L. W., Huchra, J. P., Geller, M. J., O'Brien, P., and Wilson, R. 1988 Ap. J., 326, 101 .

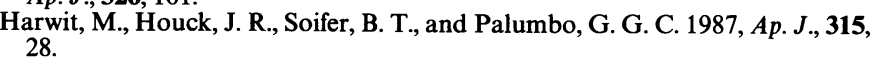

Hawkins, I., and Wright, E. L. 1988, Ap. J., 324, 46

Hayakawa, S., Matsumoto, T., Matsuo, H., Murakami, H., Sato, S., Lange, A. E., and Richards, P. L. 1987, Pub. Astr. Soc. Japan, 39, 941.

Heisler, J., and Ostriker, J. P. 1988, Ap. J., 332, 543.

Helou, G. 1989, in IAU Symposium 135, Interstellar Dust, in press.

Hildebrand, R. H. 1983, Quart. J.R.A.S., 24, 267.

Hogan, C. J. 1988, Ap. Letters, 27, 125.

Hogan, C. J., and Bond, J. R. 1988, in The Post-Recombination Universe, ed. N. Kaiser and A. N. Lasenby (Dordrecht: Kluwer), p. 141.

Houck, J. R., Schneider, D. P., Danielson, G. E., Beichman, C. A., Lonsdale, C. J., Neugebauer, G., and Soifer, B. T. 1985, Ap. J. (Letters), 290 , L5.

Johnson, D. G., and Wilkinson, D. T. 1987, Ap. J. (Letters), 313, L1.

Joseph, R. D. 1987, in Comets to Cosmology, ed. A. Lawrence (Berlin: Springer), p. 234.

Joseph, R. D., and Wright, G. S. 1985, M.N.R.A.S., 214, 87

Joy, M., Lester, D. E., Harvey, P. M., and Frueh, M. 1986, Ap. J., 307, 110.

Kaiser, N., and Silk, J. 1986, Nature, 324, 529.

Kawasaki, M., and Sato, K. 1987, Pub. Astr. Soc. Japan, 39, 837.

Kirshner, R. P., Oemler, A, Jr., Schechter, P. L., and Shectman, S. A. 1983, A.J., 88,1285

Kogut, A., et al. 1988, Ap. J., 325, 1.

Koo, D. C. 1986, in Spectral Evolution of Galaxies, ed. C. Chiosi and A. Renzini (Dordrecht: Reidel), p. 419.

Kreysa, E., and Chini, R. 1989, in Astronomy, Cosmology and Fundamental Physics, ed. M. Caffo, R. Fanti, G. Giacomelli, and A. Renzini (Dordrecht: Kluwer), p. 433.

Lacey, C. G., and Field, G. B. 1988, Ap. J. (Letters), 330, L1.
Lange, A. 1989, private communication.

Larson, G. R., and Tinsley, B. M. 1978, Ap. J., 219, 46.

Larson, R. B. 1986a, M.N.R.A.S., 218, 409.

. 1986b, in Stellar Populations, ed. C. A. Norman, A. Renzini, and M.

Tosi (Cambridge: Cambridge University Press), p. 101

$1987 a$, in Starburst and Galaxy Evolution, ed. T. Montmerle (Paris: Editions Frontieres), p. 467.

1987b, Comm. Ap., 11, 273.

1988, in Galactic and Extragalactic Star Formation, ed. R. E. Pudritz and M. Fich (Dordrecht: Kluwer), p. 459

Lequeux, J., Maucherat-Joubert, M., Deharveng, J. M., and Kunth, D. 1981, Astr. Ap., 103, 305.

Lilly, S. J. 1988, Ap. J., 333, 161.

Melaney, R. A., and Fowler, W. A. 1988, Ap. J., 333, 14.

Mather, J. C. 1982, Opt. Engineering, 21, 769.

Matsumoto, T., Akiba, M., and Murakami, H. 1987, in IAU Symposium 124, Observational Cosmology, ed. A. Hewitt, G. Burbidge, and L. Z. Fang (Dordrecht: Reidel), p. 69.

. 1988, Ap.J., 332, 575.

Matsumoto, T., Hayakawa, S., Matsuo, H., Murakami, H., Sato, S., Lange, A. E., and Richards, P. L. 1988, Ap. J., 329, 567.

Mattig, W. 1958, Astr. Nach., 284, 109.

McCarthy, P., et al. 1990 , in preparation.

McCarthy, P. J., Dickinson, M., Filippenko, A. V., Spinrad, H., and van Breugel, W. J. M. 1988, Ap. J. (Letters), 328, L29.

McDowell, J. C. 1986, M.N.R.A.S., 223, 763.

1988, in The Post-Recombination Universe, ed. N. Kaiser and A. N. Lasenby (Dordrecht: Kluwer), p. 159.

Meier, D. L., and Terlevich, R. 1981, Ap. J. (Letters), 246, L109.

Meyer, D. M., Roth, K. C., and Hawkins, I. 1989, Ap. J. (Letters), 343, L1.

Negroponte, J. 1986, M.N.R.A.S., 22, 19

Neugebauer, G., et al. 1984, Ap. J. (Letters), 278, L1.

Norman, C., and Scoville, N. 1988, Ap. J., 332, 124

O'Connell, R. W. 1986, in Stellar Populations, ed. C. A. Norman, A. Renzini, and M. Tosi (Cambridge: Cambridge University Press), p. 167.

. 1988, in Starbursts and Galaxy Evolution, ed. T.X. Thuan, T. Mountmerle, and J. Tran Thanh van (Paris: Editions Frontieres), p. 367.

Oke, J. B. 1988, in High Redshift and Primeval Galaxies, ed. J. Bergeron et al. (Paris: Editions Frontiers), p. 19

Ostriker, J. P., and Heisler, J. 1984, Ap. J., 278, 1.

Palmieri, T. M., Burginyon, G. A., Groder, R. J., Hill, R. W., Seward, F. D., and Stoering, F. D. 1971, Ap. J., 169, 33.

Partridge, R. B. 1987, in IAU Symposium 124, Observational Cosmology, ed. A. Hewitt, C. Burbidge, and L. Z. Fang (Dordrecht: Reidel), p. 31.

Peebles, P. J. E. 1986, Nature, 321, 27.

Raffelt, G., Dearborn, D., and Silk, J. 1989, Ap. J., 336, 61.

Rees, M. J., and Ostriker, J. P. 1977, M.N.R.A.S., 179, 541.

Renzini, A. 1986, in Stellar Populations, ed. C. A. Norman, A. Renzini, and M. Tosi (Cambridge: Cambridge University Press), p. 213.

Renzini, A., and Buzzoni, A. 1986, in Spectral Evolution of Galaxies, ed. C. Chiosi and A. Renzini (Dordrecht: Reidel), p. 135.

Rephaeli, Y., and Smoot, G. 1988, in Dark Matter, ed. J. Audouze and J. T. T. Van (Gif sur Yvette: Editions Frontières), p. 123.

Rice, W., Lonsdale, C. J., Soifer, B. T., Neugebauer, G., Koplan, E. L., Lloyd, L. A., de Jong, T., and Habing, H. J. 1988, Ap. J. Suppl., 68, 91.

Rieke, G. H. 1988, in Galactic and Extragalactic Star Formation, ed. R. E. Pudritz and M. Fich (Dordrecht: Kluwer), p. 561.

Rieke, G. H., Cutri, R. M., Black, J. H., Kailey, W. F., McAlary, C. W., Lebofsky, M. J., and Elston, R. 1985, Ap. J., 290, 116

Rieke, G. H., Lebofsky, M. J., Thompson, R. J., Low, F. J., and Tokunaga, A. T. 1980, Ap. J., 238, 24.

Rowan-Robinson, M 1986, M N R A S 219, 737.

Rowan-Robinson, M., and Carr, B. 1988, in The Post-Recombination Universe, ed. N. Kaiser, and A. N. Lasenby (Dordrecht: Kluwer), p. 125.

Sale, K. E., and Mathews, G. J. 1986, Ap. J.(Letters), 309, L1.

Sandage, A. 1986, Ann. Rev. Astr. Ap., 24, 421. 1987, in The Galaxy, ed. G. Gilmore and B. Carswell (Dordrecht: Reidel), p. 321

Sandage, A., and Fouts, G. 1987, A.J., 93, 74

Sanders, D. B., Soifer, B. T., Elias, J. H., Madore, B. F., Matthews, K., Neugebauer, G., and Scoville, N. Z. 1988a, Ap. J., 325, 74 .

Sanders, D. B., Soifer, B. T., Elias, J. H., Neugebauer, G., and Matthews, K. $1988 b$, Ap. J. (Letters), 328, L35.

Sarajedini, A., and King, C. R. 1988, preprint

Scalo, J. 1986, Fund Cosmic. Phys., 11, 1.

Schaaf, R., Pietsch, W., Biermann, P. L., Kronberg, P. P., and Schmutzler, T. 1989, Ap. J., 336, 722 .

Schmidt, M., and Green, R. F. 1986, Ap. J., 305, 68

Schmidt, M., Gunn, J. E., and Schneider, D. 1989, in preparation.

Schwartz, D. A., and Tucker, W. H. 1988, Ap. J., 332, 157.

Schweizer, F. 1987, in Nearly Normal Galaxies, ed. S. Faber (New York: Springer), p. 18.

Scoville, N. 1988, in Galactic and Extragalactic Star Formation, ed. R, E. Pudritz and M. Fich (Dordrecht: Kluwer), p. 541.

Setti, G., and Woltjer, L. 1982, in Astrophysical Cosmology, ed. H. A. Bruck, G. V. Coyne, and M. S. Longair (Vatican City: Pontificia Academia Scientiarum), p. 315

Shapiro, P. R., and Kang, H. 1987, Ap. J., 318, 32. 
Shapiro, S. L., and Teukolsky, S. A. 1983, Black Holes, White Dwarfs, and Neutron Stars: The Physics of Compact Objects (New York: Wiley).

Silk, J., 1987a, in IAU Symposium 124, Observational Cosmology, ed. A. Hewitt, G. Burbidge, and L. Z. Fang (Dordrecht: Reidel), p. 391.

. $1987 b$, in IAU Symposium 117 Dark Matter in the Universe, ed. J. Kormendy and G. R. Knapp (Dordrecht: Reidel), p. 335.

1987c, in IAU Symposium 115, Star-Forming Regions, ed. M. Peimbert

and J. Jugaku (Dordrecht: Reidel), p. 663.

1988a, in Galactic and Extragalactic Star Formation, ed. R. E. Pudritz and M. Fich (Dordrecht: Kluwer), p. 503.

1988b, in Dark Matter, ed. J. Audouze and J. T. T. Van (Gif sur Yvette:

Editions Frontières), p. 3

1989 , private communication

Silk, J., and Norman, C. 1981, Ap. J., 247, 59.

Silk, J., and Szalay, A. 1987, Ap. J. (Letters), 323, L107.

Silk, J., and Tamanaha, 1989, in preparation.

Smith, J., Gehrz, R. D., Grasdalen, G. L., Hackwell, J. A., and Dietz, R. D. 1988, Ap. J., 329, 107.

Smoot, G. F., Bensadoun, M., Bersanelli, M., De Amici, G., Kogut, A., Levin, S., and Witebsky, C. 1987, Ap. J. (Letters), 317, L45.

Smoot, G. F., Levin, S. M., Witebsky, C., De Amici, G., and Rephaeli, Y. 1988, Ap. J., 331, 653.

Soifer, B. T., et al. 1984, Ap. J. (Letters), 283, L1.

Soifer, B. T., Houck, J. R., and Neugebauer, G. 1987, Ann. Rev. Astr. Ap., 25, 187.

Spinrad, H. 1987, in High Redshift and Primeval Galaxies, ed. J. Bergeron, D. Kunth, B. Rocca-Volmerange, and J. Tran Thanh Van (Paris: Editions Frontieres), p. 59.

S. DJoRgovski: Mailstop 105-24, Caltech, Pasadena, CA 91125 SPAN:6035::george]

N. WeIR: Mailstop 105-24, Caltech, Pasadena, CA 91125

[SPAN:6035::weir]
Sunyaev, R. A., and Zel'dovich, Y. B. 1980, Ann. Rev. Astr. Ap., 18, 537.

Taylor, G., and Wright, E. 1989, Ap. J., 339, 619.

Telesco, C. M., and Harper, D. A. 1980, Ap. J., 235, 392.

Thronson, H. T., and Harper, D. A. 1979, Ap. J., 230, 133.

Tyson, J. A. 1988a, A.J., 96, 1 . Kron and A. Renzini (Dordrecht: Kluwer), p. 187.

VandenBerg, D. A. 1988, in The Extragalactic Distance Scale, ed. S. van den Bergh and C. J. Pritchet (Provo: Bingham Young University Print Services), p. 187.

Watson, M. G., Stanger, V., and Griffiths, R. E. 1984, Ap. J., 286, 144.

Weedman, D. W. 1987, in Star Formation in Galaxies, ed. C. J. Lonsdale Persson (Washington, D.C.: NASA CP-2466).

Weedman, D. W., Feldman, F. R., Balzano, V. A., Ramsey, L. W., Sramek R. A., and Wu, C.-C. 1981, Ap. J., 248, 105 .

Werner, M. W., and Eisenhardt, P. 1988, Ap. Letters, $27,89$.

White, S. D. M., and Rees, M. J. 1978, M.N.R.A.S., 183, 341.

Winget, D. E., Hansen, C. J., Liebert, J., Van Horn, H. M., Fontaine, G., Nathen, R. E., Kepler, S. O., and Lamb, D. Q. 1987, Ap. J. (Letters), 315, L77.

Wright, E. L. 1987, Ap. J., 320, 818.

Yang, J., Turner, M. S., Steigman, G., Schramm, D. N., and Olive, K. A. 1984, Ap. J., 281, 493.

Zinn, R. 1986, in Stellar Populations, ed. C. A. Norman, A. Renzini, and M Tosi (Cambridge: Cambridge University Press), p. 73. 